\title{
The geometry of surface-by-free groups
}

\author{
Benson Farb* and Lee Mosher ${ }^{\dagger}$
}

August 29, 2000

\begin{abstract}
We show that every word hyperbolic, surface-by-(noncyclic) free group $\Gamma$ is as rigid as possible: the quasi-isometry group of $\Gamma$ equals the abstract commensurator group $\operatorname{Comm}(\Gamma)$, which in turn contains $\Gamma$ as a finite-index subgroup. As a corollary, two such groups are quasi-isometric if and only if they are commensurable, and any finitely-generated group quasi-isometric to $\Gamma$ must be weakly commensurable with $\Gamma$. We use quasi-isometries to compute $\operatorname{Comm}(\Gamma)$ explicitly, an example of how quasi-isometries can actually detect finite-index information. The proofs of these theorems involve ideas from coarse topology, Teichmüller geometry, pseudo-Anosov dynamics, and singular SOLV geometry.
\end{abstract}

\section{Introduction}

Let $\Sigma_{g}$ be a closed surface of genus $g \geq 2$, and let $\mathcal{M}\left(\Sigma_{g}\right)=\pi_{0}\left(\operatorname{Homeo}\left(\Sigma_{g}\right)\right)$ denote the mapping class group of $\Sigma_{g}$. A Schottky subgroup $H$ of $\mathcal{M}\left(\Sigma_{g}\right)$ is a free group of pseudo-Anosov mapping classes whose action on the Teichmüller space $\mathcal{T}\left(\Sigma_{g}\right)$ is "weak convex cocompact" - the group $H$ has a limit set in Thurston's boundary of $\mathcal{T}\left(\Sigma_{g}\right)$, when $\operatorname{rank}(H) \geq 2$ this limit set is a Cantor set, and the action of $H$ on the "weak convex hull" of the limit set is cocompact. Schottky subgroups of $\mathcal{M}\left(\Sigma_{g}\right)$ exist in abundance: given any collection $\left\{\phi_{1}, \ldots, \phi_{r}\right\}$ of pairwise independent pseudo-Anosov mapping classes of $\Sigma_{g}$, for any sufficiently large positive integers $a_{1}, \ldots, a_{r}$ the elements $\left\{\phi_{1}^{a_{1}}, \ldots, \phi_{r}^{a_{r}}\right\}$ freely generate a Schottky subgroup. See $\S 3$ for a review of Schottky groups, taken from [Mos97] and [FM00b].

Given $g \geq 2$ and a Schottky subgroup $H<\mathcal{M}\left(\Sigma_{g}\right) \approx \operatorname{Out}\left(\pi_{1}\left(\Sigma_{g}\right)\right)$, one can construct a group $\Gamma_{H}$ given by the split extension

$$
1 \rightarrow \pi_{1}\left(\Sigma_{g}\right) \rightarrow \Gamma_{H} \rightarrow H \rightarrow 1
$$

\footnotetext{
* Supported in part by NSF grant DMS 9704640 and by a Sloan Foundation Fellowship.

${ }^{\dagger}$ Supported in part by NSF grant DMS 9504946.
} 
The groups $\Gamma_{H}$ are precisely the surface-by-free groups which are word hyperbolic [FM00b], and the construction of [Mos97] shows that they are abundant.

We are interested in studying quasi-isometries of the groups $\Gamma_{H}$ for several reasons: the $\Gamma_{H}$ provide basic examples of rigidity theorems for word hyperbolic groups outside the context of negatively curved manifolds (see also [Bou00], [KK98]); they are examples of groups which can be viewed as phase spaces of dynamical systems arising from hyperbolic endomorphisms of manifolds (see also [FM98], [FM99], [FM00a]); and they provide examples of groups which are as rigid as possible in a very concrete sense (see Theorem 1.3 below). In particular, each $\Gamma_{H}$ has finite index in its own abstract commensurator $\operatorname{Comm}\left(\Gamma_{H}\right)$ as well as in its quasi-isometry group. The computation of $\operatorname{Comm}\left(\Gamma_{H}\right)$ given in Theorem 1.3 is explicit, one of the few instances outside lattices in Lie groups where this has been done.

Finally, we propose the general problem of studying the asymptotic geometry of extensions of surface groups $\Sigma$. These groups exhibit a beautiful and rich geometry which is encoded by a subgroup of $\mathcal{M}(\Sigma)$ acting on the Teichmüller space $\mathcal{T}(\Sigma)$. Some elements of the geometry of such groups can be found in [Mos96], [Mit98] and [FM00b], but our needs will require a somewhat involved account of this theory for extensions of surface groups by Schottky groups, which we give in $\S 4$.

\section{Statement of results}

Our first theorem gives a complete classification of the groups $\Gamma_{H}$ up to quasiisometry. Although the usual quasi-isometry invariants such as growth, ends, isoperimetric functions, etc., are the same for all of these groups, they are only quasi-isometric under the strictest algebraic conditions.

Given a Schottky subgroup $H$ of $\mathcal{M}(\Sigma)$, we will show in $\S 7.2$ that there is a smallest 2 -orbifold covered by $\Sigma$, denoted $\mathcal{O}_{H}$, such that $H$ descends via the covering map $\Sigma \rightarrow \mathcal{O}_{H}$ to a Schottky subgroup of $\mathcal{M}\left(\mathcal{O}_{H}\right)$, still denoted $H$. The orbifold $\mathcal{O}_{H}$ plays an important role in describing the quasi-isometry class of the surface-by-free group $\Gamma_{H}$.

Theorem 1.1 (Classification Theorem). Given $g_{1}, g_{2} \geq 2$, let $H_{1}<\mathcal{M}\left(\Sigma_{g_{1}}\right)$ and $H_{2}<\mathcal{M}\left(\Sigma_{g_{2}}\right)$ by Schottky subgroups of rank $\geq 2$. The following are equivalent:

(1) The surface-by-free groups $\Gamma_{H_{1}}$ and $\Gamma_{H_{2}}$ are quasi-isometric.

(2) $\Gamma_{H_{1}}$ and $\Gamma_{H_{2}}$ are abstractly commensurable, meaning that they have finiteindex subgroups which are isomorphic. 
(3) There is an isomorphism $\mathcal{O}_{H_{1}} \approx \mathcal{O}_{H_{2}}$ such that in the group $\mathcal{M}\left(\mathcal{O}_{H_{1}}\right)=$ $\mathcal{M}\left(\mathcal{O}_{H_{2}}\right)$ the Schottky subgroups $H_{1}$ and $H_{2}$ are commensurable, meaning that $H_{1} \cap H_{2}$ has finite index in each of $H_{1}$ and $H_{2}$.

(4) There is an isomorphism $\mathcal{O}_{H_{1}} \approx \mathcal{O}_{H_{2}}$ such that in the group $\mathcal{M}\left(\mathcal{O}_{H_{1}}\right)=$ $\mathcal{M}\left(\mathcal{O}_{H_{2}}\right)$ the Schottky subgroups $H_{1}$ and $H_{2}$ have the same limit set in the Thurston boundary of the Teichmüller space $\mathcal{T}\left(\mathcal{O}_{H_{1}}\right)=\mathcal{T}\left(\mathcal{O}_{H_{2}}\right)$.

The next theorem shows that each of the groups $\Gamma_{H}$ is determined among all finitely-generated groups by its asymptotic geometry, up to finite data.

Theorem 1.2 (Quasi-isometric rigidity). Let $\Gamma_{H}$ be a surface-by-free group with $H<\mathcal{M}\left(\Sigma_{g}\right)$ a Schottky group of rank $\geq 2$. If $G$ is any finitely-generated group which is quasi-isometric to $\Gamma_{H}$, then there is a finite normal subgroup $F<G$ such that $G / F$ is abstractly commensurable to a surface-by-free group. Combining with Theorem 1.1 it follows that $G / F$ is abstractly commensurable to $\Gamma_{H}$.

Remark. We emphasize that it is essential for our methods that $\operatorname{rank}(H) \geq 2$ in the statements of Theorems 1.1 and 1.2. Indeed, when $H$ is a rank 1 Schottky subgroup, in other words an infinite cyclic subgroup generated by a pseudo-Anosov mapping class, then $\Gamma_{H}$ is the fundamental group of a closed hyperbolic 3-manifold that fibers over the circle [Ota96], and hence all the groups $\Gamma_{H}$ with $H$ Schottky of rank 1 are quasi-isometric to each other and to $\mathbf{H}^{3}$. Moreover, the restatement of Theorem 1.2 (minus the last sentence) for $H$ of rank 1 is equivalent to Thurston's virtual surface bundle conjecture for closed hyperbolic 3-manifolds. ${ }^{1}$

Theorem 1.2 and (most of) Theorem 1.1 follow from our main result, Theorem 1.3 below.

Commensurations and quasi-isometries. Recall that a commensuration of $\Gamma$ is an isomorphism between finite index subgroups of $\Gamma$. Composition of two commensurations is defined on a further finite index subgroup. Two commensurations are equivalent if they agree on a common finite index subgroup. Composition of equivalence classes gives a well-defined group operation, and we thereby obtain the abstract commensurator group $\operatorname{Comm}(\Gamma)$ of the group $\Gamma$.

The quasi-isometry group $\mathrm{QI}(\Gamma)$ is the group of coarse equivalence classes of self quasi-isometries of $\Gamma$ (endowed with any word metric), where two quasi-isometries are coarsely equivalent if they have finite distance in the sup norm.

\footnotetext{
${ }^{1}$ This equivalence, however, hides the following fact: Theorem 1.1 provides a commensuration from $\Gamma_{H_{1}}$ to $\Gamma_{H_{2}}$ coarsely taking $\pi_{1}\left(\Sigma_{g_{2}}\right)$ to $\pi_{1}\left(\Sigma_{g_{2}}\right)$, a situation which is often impossible for rank 1 ; see $\S 8.2$. Hence the proofs of Theorems 1.1 and 1.2 cannot shed light on the virtual surface bundle conjecture.
} 
In general it is difficult to compute the abstract commensurator of a group. The computation for irreducible lattices in semisimple groups $G \neq \operatorname{PSL}(2, \mathbf{R})$ is the content of Mostow Rigidity together with theorems of Borel and Margulis (see e.g. [Zim84]). Our main result, Theorem 1.3, says that the groups $\operatorname{Comm}\left(\Gamma_{H}\right)$ and $\mathrm{QI}\left(\Gamma_{H}\right)$ are isomorphic, and gives an explicit computation of these groups. This is the first time we know of where quasi-isometries are used to compute the abstract commensurator group. While our expression for $\operatorname{Comm}\left(\Gamma_{H}\right)$ is purely algebraic, we do not know how to do the computation algebraically.

Recall that given groups $K<Q$, the (relative) commensurator of $K$ in $Q$, denoted $\operatorname{Comm}_{Q}(K)$, is the subgroup of all $q \in Q$ such that conjugation by $q$ takes some finite index subgroup of $K$ to another, or equivalently $K \cap q K q^{-1}$ has finite index in both $K$ and $q K q^{-1}$; this subgroup is also known as the virtual normalizer of $K$ in $Q$. The group $\mathcal{C}=\operatorname{Comm}_{\mathcal{M}\left(\mathcal{O}_{H}\right)}(H)$, the relative commensurator of $H$ in $\mathcal{M}\left(\mathcal{O}_{H}\right)$, plays a key role in computing the abstract commensurator of $\Gamma_{H}$ :

Theorem 1.3 (Computation of $\mathrm{QI}\left(\Gamma_{H}\right)$ and $\operatorname{Comm}\left(\Gamma_{H}\right)$ ). Given $g \geq 2$ and a Schottky group $H<\mathcal{M}\left(\Sigma_{g}\right)$ of rank $\geq 2$, the natural homomorphism

$$
\operatorname{Comm}\left(\Gamma_{H}\right) \rightarrow \mathrm{QI}\left(\Gamma_{H}\right)
$$

is an isomorphism. Furthermore, these groups are isomorphic to the group $\Gamma_{\mathcal{C}}$ given explicitly by the short exact sequence

$$
1 \rightarrow \pi_{1}\left(\mathcal{O}_{H}\right) \rightarrow \Gamma_{\mathcal{C}} \rightarrow \operatorname{Comm}_{\mathcal{M}\left(\mathcal{O}_{H}\right)}(H) \rightarrow 1
$$

Moreover, $\mathcal{C}=\operatorname{Comm}_{\mathcal{M}\left(\mathcal{O}_{H}\right)}(H)$ contains $H$ as a finite index subgroup. In particular, $\Gamma_{H}$ has finite index in $\operatorname{Comm}\left(\Gamma_{H}\right) \approx \mathrm{QI}\left(\Gamma_{H}\right)$.

This theorem is proved in Sections 7.3 and 7.4.

The fact that $\Gamma_{H}$ has finite index in $\mathrm{QI}\left(\Gamma_{H}\right)$ and also in $\operatorname{Comm}\left(\Gamma_{H}\right)$ shows that the groups $\Gamma_{H}$ are extremely rigid. Among irreducible lattices in semisimple Lie groups, this phenomenon holds for the noncocompact, nonarithmetic lattices and for no other lattice; see [Mar91] for the commensurator statement, and [Sch96] and e.g. [Far97] for the quasi-isometry statement.

\section{Outline of the proof of Theorem 1.3}

After some preliminary material, we begin in $\S 4$ by constructing a quasi-isometric model space $X_{H}$ on which $\Gamma_{H}$ acts properly cocompactly by isometries. A Schottky subgroup $H<\mathcal{M}(\Sigma)$ gives an $H$-equivariant embedding of the Cayley graph $T_{H}$ of $H$, a tree, in the Teichmüller space $\mathcal{T}(\Sigma)$. This embedding gives a $\Sigma$-bundle over $T_{H}$, each fiber carrying a hyperbolic structure representing the point of $T_{H} \subset \mathcal{T}(\Sigma)$ 
over which that fiber lies. The universal cover of this $\Sigma$-bundle is our model space $X_{H}$, an $\mathbf{H}^{2}$-bundle over the tree $T_{H}$.

An isometry of $\Gamma_{H}$ acts on $X_{H}$, permuting or "respecting" various patterns of geometric objects, typically foliations. Indeed, the same patterns respected by isometries of $X_{H}$ are also respected by the finite index supergroup $\Gamma_{\mathcal{C}}$ which is defined by the short exact sequence given in Theorem 1.3; see $\S 7.3$ for the precise definition of $\Gamma_{\mathcal{C}}$.

The rest of the proof is devoted to showing that an arbitrary quasi-isometry $f: X_{H} \rightarrow X_{H}$ coarsely respects so many patterns that it must be close to an element of $\Gamma_{\mathcal{C}}$. Using coarse topology, we show in $\S 4$ that $f$ permutes the collection of "hyperplanes" $P_{w}$; these are the $\mathbf{H}^{2}$-bundles over bi-infinite lines $w$ in $T_{H}$. The Schottky property is used to relate each line $w$ to a Teichmüller geodesic, which in turn allows us to impose extra structure on the hyperplane $P_{w}$ : a "pseudo-Anosov flow" and a singular SOLV structure. In $\S 5$ we use this structure to prove that $f$ coarsely respects several dynamically defined foliations associated to these flows, such as the stable and unstable foliations.

In $\S 6$, we apply the above together with R. Schwartz's geodesic pattern rigidity [Sch97] to show that the quasi-isometry $f$ actually permutes the collection of periodic hyperplanes, i.e. those $P_{w}$ with $w$ a bi-infinite periodic line in the tree $T_{H}$.

In $\S 7$ we study how the quasi-isometry $f$ acts on the limit set of $H$ in Thurston's boundary of $\mathcal{T}(\Sigma)$, the space of projective measured foliations. This limit set is a Cantor set, and the set of periodic hyperplanes gives a countable dense subset which is preserved by the action of $f$. This information is then used to show that $f$ is close to an element of $\Gamma_{\mathcal{C}}$.

The final parts of the proofs of all of the main theorems are contained in $\S 7$.

Surfaces versus orbifolds. Our main theorems are stated solely for closed, oriented surfaces $\Sigma_{g}$ of genus $g \geq 2$, and Schottky subgroups $H$ of $\mathcal{M}\left(\Sigma_{g}\right)$. But the conclusions of these theorems force us to consider a wider realm: closed 2-orbifolds $\mathcal{O}$ and virtual Schottky subgroups of $\mathcal{M}(\mathcal{O})$; see e.g. Theorem 1.3 in which, as the proof will show, $\operatorname{Comm}_{\mathcal{M}\left(\mathcal{O}_{H}\right)}(H)$ is a virtual Schottky subgroup of $\mathcal{M}\left(\mathcal{O}_{H}\right)$. Various results that we will need about (virtual) Schottky subgroups of orbifold mapping class groups are formulated in [FM00b].

This raises the question of whether the quasi-isometry classes of (orbifold)by-(virtual Schottky) groups constitute a wider universe than the quasi-isometry classes of (surface)-by-(Schottky) groups. The answer is no: these universes are identical. The proof is given in Section 8.1. 
Acknowledgements We are grateful to Kevin Whyte for crucial help in proving Theorem 7.4.

\section{Preliminaries}

In this section we briefly review some facts about Teichmüller space and about quasi-isometries. For details we refer to [Abi80], [FLP $\left.{ }^{+} 79\right]$, [IT92].

For most of the paper, while we concentrate on the proof of Theorem 1.3, we shall fix the genus $g$ and denote $\Sigma=\Sigma_{g}$.

\subsection{Teichmüller space and mapping class groups}

The Teichmüller space of $\Sigma$, denoted $\mathcal{T}=\mathcal{T}(\Sigma)$, has two equivalent descriptions, related to each other by Riemann's uniformization theorem: $\mathcal{T}$ is the space of conformal structures on $\Sigma$ modulo isotopy; or it is the space of hyperbolic structures on $\Sigma$ modulo isotopy. A topology and a real analytic structure on $\mathcal{T}$ is specified by the geodesic length embedding $\mathcal{T} \rightarrow[0, \infty)^{\mathcal{C}}$ where $\mathcal{C}$ is the set of isotopy classes of nontrivial simple closed curves on $\Sigma$, and a hyperbolic structure on $\Sigma$ determines an element of $[0, \infty)^{\mathcal{C}}$ by taking the length of the unique closed geodesic in each isotopy class. With respect to this topology, $\mathcal{T}$ is homeomorphic to Euclidean space of dimension $6 g-6$.

The mapping class group of $\Sigma$, denoted $\mathcal{M}=\mathcal{M}(\Sigma)$, is the group $\pi_{0}(\operatorname{Homeo}(\Sigma)=$

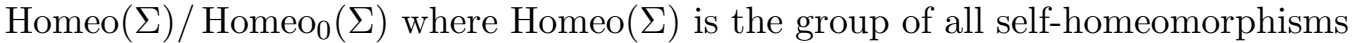
of $\Sigma$ and $\mathrm{Homeo}_{0}(\Sigma)$ is the normal subgroup of self-homeomorphisms isotopic to the identity.

The group $\mathcal{M}$ acts real analytically on $\mathcal{T}$. Also, $\mathcal{M}$ acts on $\mathcal{C}$ and so on $[0, \infty)^{\mathcal{C}}$, and the embedding $\mathcal{T} \rightarrow[0, \infty)^{\mathcal{C}}$ is $\mathcal{M}$-equivariant. The action of $\mathcal{M}$ on $\mathcal{T}$ is properly discontinuous. The quotient orbifold $M=M(\Sigma)=\mathcal{T} / \mathcal{M}$ is called the moduli space of $\Sigma$. A subset of $\mathcal{T}$ is said to be cobounded if its image under the quotient map $\mathcal{T} \rightarrow \mathcal{M}$ is bounded.

\subsection{Measured foliations}

A measured foliation on $\Sigma$ is a foliation on the complement of a finite set of singularities, together with a positive, transverse Borel measure, such that each singularity is an $n$-pronged singularity for some $n \geq 3$, locally modelled on the horizontal measured foliation of the quadratic differential $z^{n-2} d z$ in the complex plane. A saddle connection of a measured foliation is leaf segment which connects two distinct singularities, and Whitehead equivalence is the equivalence relation on the set of measured foliations generated by isotopy and the collapse of saddle 
connections. The measured foliation space of $\Sigma$, denoted $\mathcal{M} F=\mathcal{M} F(\Sigma)$, is the space of Whitehead equivalence classes of measured foliations on $\Sigma$. A topology on $\mathcal{M F}$ is specified by the "transverse measure embedding" $\mathcal{M F} \rightarrow[0, \infty)^{\mathcal{C}}$, where a measured foliation determines an element of $[0, \infty)^{\mathcal{C}}$ by taking the infimum of the transverse measures of representatives of $\mathcal{C}$.

Given a measured foliation $\mathcal{F}$ and $r \in(0, \infty)$, multiplying the transverse measure on $\mathcal{F}$ by $r$ gives a new measured foliation denoted $r \mathcal{F}$. This gives a free action of $(0, \infty)$ on $\mathcal{M} F$, whose quotient space is the space of projective measured foliations on $\Sigma$, denoted $\mathbf{P} \mathcal{M} F=\mathbf{P} \mathcal{M} F(\Sigma)$.

The embedding $\mathcal{T} \hookrightarrow[0, \infty)^{\mathcal{C}}$, composed with the projectivization map $[0, \infty)^{\mathcal{C}} \rightarrow$ $\mathbf{P}[0, \infty)^{\mathcal{C}}$, produces an embedding $\mathcal{T} \hookrightarrow \mathbf{P}[0, \infty)^{\mathcal{C}}$. The embedding $\mathcal{M} F \hookrightarrow[0, \infty)^{\mathcal{C}}$ induces an embedding $\mathbf{P} \mathcal{M} F \hookrightarrow \mathbf{P}[0, \infty)^{\mathcal{C}}$. Thurston's Compactification Theorem $\left[\mathrm{FLP}^{+} 79\right]$ says that image of $\overline{\mathcal{T}}=\mathcal{T} \cup \mathbf{P} \mathcal{M} F$ in $\mathbf{P}[0, \infty)^{\mathcal{C}}$ is a closed ball of dimension $6 g-6$, whose interior is $\mathcal{T}$ and whose boundary sphere is $\mathbf{P} \mathcal{M} F$.

\section{$2.3 \quad$ Geodesics in $\mathcal{T}$}

The Teichmüller metric and its geodesics are usually described in terms of holomorphic quadratic differentials on Riemann surfaces. Using results of Gardiner and Masur [GM91] and of Hubbard and Masur [HM79], the metric can be presented directly in terms of measured foliations.

Consider of pair of measured foliations $\mathcal{F}_{x}, \mathcal{F}_{y}$ which are transverse, meaning that they have the same singular set, at each singularity $\mathcal{F}_{x}$ and $\mathcal{F}_{y}$ have the same number of prongs, they are transverse in the usual sense away from the singularities, and near an $n$-pronged singularity they are locally modelled on the horizontal and vertical measured foliations of the quadratic differential $z^{n-2} d z$ on the complex plane. Let $|d y|,|d x|$ denote the transverse measures on $\mathcal{F}_{x}, \mathcal{F}_{y}$, respectively; the leaves of $\mathcal{F}_{x}$ should be visualized as horizontal lines with transverse measure $|d y|$, and the leaves of $\mathcal{F}_{y}$ as vertical lines with transverse measure $|d x|$. The formula $d x^{2}+d y^{2}$ defines a singular Euclidean metric on $\Sigma$ denoted $\mu\left(\mathcal{F}_{x}, \mathcal{F}_{y}\right)$, with total area

$$
\operatorname{Area}(\mu)=\int_{\Sigma}|d x||d y|<\infty
$$

Underlying the metric $\mu\left(\mathcal{F}_{x}, \mathcal{F}_{y}\right)$ is a conformal structure on the complement of the singularities, but the singularities are removable and so we obtain a conformal structure on $\Sigma$ and a point in $\mathcal{T}$ denoted $\sigma\left(\mathcal{F}_{x}, \mathcal{F}_{y}\right)$. This gives a well-defined map from a certain subset of $\mathcal{M} F \times \mathcal{M} F$ to $\mathcal{T}$. Namely, letting $\mathcal{U} \subset \mathcal{M} F \times \mathcal{M} F$ denote the set of pairs $(\xi, \eta)$ which are represented by a transverse pair $\left(\mathcal{F}_{x}, \mathcal{F}_{y}\right)$ of measured foliations, it follows that $\sigma(\xi, \eta)=\sigma\left(\mathcal{F}_{x}, \mathcal{F}_{y}\right)$ is well-defined independent 
of the choice of the representative pair $\left(\mathcal{F}_{x}, \mathcal{F}_{y}\right)$, defining a map $\mathcal{U} \rightarrow \mathcal{T}$ (see [GM91], Theorem 3.1).

A transverse pair $\mathcal{F}_{x}, \mathcal{F}_{y}$ is normalized if $\operatorname{Area}\left(\mu\left(\mathcal{F}_{x}, \mathcal{F}_{y}\right)\right)=1$. Let $\mathcal{U}_{0} \subset \mathcal{U}$ be the subset represented by normalized transverse pairs. For each $\left(\mathcal{F}_{x}, \mathcal{F}_{y}\right) \in \mathcal{U}_{0}$, the map $t \mapsto \gamma(t)=\sigma\left(e^{t} \mathcal{F}_{x}, e^{-t} \mathcal{F}_{y}\right)$ is a real analytic embedding of $\mathbf{R}$ in $\mathcal{T}$; the image of this embedding depends only on the projective classes $\xi=\mathbf{P} \mathcal{F}_{x}, \eta=\mathbf{P} \mathcal{F}_{y}$ and is denoted $\overleftrightarrow{(\xi, \eta)}=\overleftrightarrow{\left(\mathbf{P} \mathcal{F}_{x}, \mathbf{P} \mathcal{F}_{y}\right)}$. Teichmüller's Theorem [Abi80] says that any two points $p \neq q \in \mathcal{T}$ are contained in a unique such line $\overleftrightarrow{\left(\mathbf{P} \mathcal{F}_{x}, \mathbf{P} \mathcal{F}_{y}\right)}$; moreover if $s, t \in \mathbf{R}$ are such that $p=\sigma\left(e^{t} \mathcal{F}_{x}, e^{-t} \mathcal{F}_{y}\right)$ and $q=\sigma\left(e^{s} \mathcal{F}_{x}, e^{-s} \mathcal{F}_{y}\right)$, then the formula

$$
d(p, q)=|s-t|
$$

gives a well-defined metric on $\mathcal{T}$, known as the Teichmüller metric. Each line $\overleftrightarrow{(\xi, \eta)}$ then becomes a bi-infinite geodesic in $\mathcal{T}$. If we restrict the parameterization $\gamma(t)=\sigma\left(e^{t} \mathcal{F}_{x}, e^{-t} \mathcal{F}_{y}\right)$ to the half-line $t \in[0, \infty)$ then we obtain a geodesic ray in $\mathcal{T}$ and we call the point $\mathbf{P} \mathcal{F}_{x} \in \mathbf{P} \mathcal{M} F$ the ending foliation of the ray; if $\sigma=\gamma(0)$ and $\xi=\mathbf{P} \mathcal{F}_{x}$ then this ray is denoted $\overrightarrow{[\sigma, \xi)}$. For any Teichmüller line $\overleftrightarrow{(\xi, \eta)}$ the two points $\xi, \eta$ are called the ending foliations of the line. Any two points $\sigma, \tau \in \mathcal{T}$ are the endpoints of a unique finite geodesic segment, denoted $\overline{\sigma \tau}$.

With respect to the Teichmüller metric, $\mathcal{T}$ is a complete metric space on which $\mathcal{M}$ acts by isometries. Royden's Theorem [Roy71] says, when the surface $\Sigma$ is closed and oriented, that the homomorphism $\mathcal{M} \rightarrow \operatorname{Isom}(\mathcal{T})$ is an isomorphism, except for a small kernel on certain small surfaces: on $\Sigma_{2}$ the single nontrivial element of the kernel being the hyperelliptic involution of $\Sigma_{2}$.

Given $\sigma \in \mathcal{T}$ and $\xi \in \mathbf{P} \mathcal{M} F$ there is exactly one ray in $\mathcal{T}$ with endpoint $\sigma$ and with ending foliation $\xi$; we denote this ray $\overrightarrow{[\sigma, \xi)}$. This gives a one-to-one correspondence between $\mathcal{T} \times \mathbf{P} \mathcal{M} F$ and geodesic rays in $\mathcal{T}$. Given $\xi, \eta \in \mathbf{P} \mathcal{M} F$, there exists at most one geodesic in $\mathcal{T}$ with ending foliations $\xi, \eta$, and it exists if and only if $(\xi, \eta)$ is in the image of $\mathcal{U}_{0} \subset \mathcal{M} F \times \mathcal{M} F$ under the projectivization map $\mathcal{M} F \times \mathcal{M} F \rightarrow \mathbf{P} \mathcal{M} F \times \mathbf{P} \mathcal{M} F$. If this geodesic exists we denote it $\overleftrightarrow{(\xi, \eta)}$ This gives a one-to-one correspondence between a certain subset of $\mathbf{P} \mathcal{M} F \times \mathbf{P} \mathcal{M} F$ and the set of geodesics in $\mathcal{T}$.

Remark. It is not in general true that the end of the ray $\overrightarrow{[\sigma, \xi)}$ converges in $\overline{\mathcal{T}}$ to the point $\xi$; however, it is at least true for cobounded rays.

Let $T \mathcal{T}$ denote the tangent space of $\mathcal{T}$. There is an embedding $\mathcal{T} \times \mathbf{P} \mathcal{M} F \mapsto$ $T \mathcal{T}$, taking $(\sigma, \xi)$ to the tangent vector at $\sigma$ of the ray $\overrightarrow{[\sigma, \xi)}$ in $T \mathcal{T}$, denoted $D \overrightarrow{[\sigma, \xi)}$. The image of this embedding will be denoted $T^{1} \mathcal{T}$, called the unit tangent bundle of $\mathcal{T}$, and $T^{1} \mathcal{T}$ is, in fact, a topological sphere bundle over $\mathcal{T}$. Moreover, the map 
$\mathcal{U}_{0} \rightarrow T^{1} \mathcal{T}$ taking $(\xi, \eta)$ to $D \overrightarrow{[\sigma(\xi, \eta), \eta)}$, is a homeomorphism. See [GM91] and [HM79] for proofs.

Remark. The Teichmüller metric is not a Riemannian metric, although it is a Finsler metric. As such, the unit tangent sphere $T_{\sigma}^{1} \mathcal{T}$ at each $\sigma \in \mathcal{T}$ is not a true ellipsoid in the vector space $T_{\sigma} \mathcal{T}$, but instead a more general convex, centrally symmetric sphere [Roy71].

The flow on $\mathcal{U}_{0}$ defined by $(\xi, \eta) \cdot t=\left(e^{t} \xi, e^{-t} \eta\right)$ pushes forward under the homeomorphism $\mathcal{U}_{0} \rightarrow T^{1} \mathcal{T}$ to a flow on $T^{1} \mathcal{T}$, namely the geodesic flow of $\mathcal{T}$. In other words, each tangent vector $v \in T^{1} \mathcal{T}$ is tangent to a unique geodesic $\gamma$, and the geodesic flow $v \cdot t$ is obtained by pushing $v$ forward a distance $t$ along $\gamma$.

\subsection{Pseudo-Anosov homeomorphisms}

A homeomorphism $h: \Sigma \rightarrow \Sigma$ is pseudo-Anosov if there exists a transverse pair of measured foliations $\mathcal{F}_{x}, \mathcal{F}_{y}$ and a $\lambda>1$ such that $h\left(\mathcal{F}_{x}\right)=\lambda \mathcal{F}_{x}$ and $h\left(\mathcal{F}_{y}\right)=$ $\lambda^{-1} \mathcal{F}_{y}$; the foliations $\mathcal{F}_{x}, \mathcal{F}_{y}$ are called the stable and unstable measured foliations of $h$, and $\lambda$ is the expansion factor of $h$. A mapping class $H \in \mathcal{M}$ is said to be pseudo-Anosov if and only if it has a representative $h: \Sigma \rightarrow \Sigma$ which is pseudoAnosov.

By construction, a mapping class $H \in \mathcal{M}=\operatorname{Isom}(\mathcal{T})$ is pseudo-Anosov if and only if there exists a geodesic $\gamma$ in $\mathcal{T}$ such that $H(\gamma)=\gamma$ and the action of $H$ on $\gamma$ is a translation of nonzero length. In this case, the geodesic $\gamma$ is unique and is called the axis of $H$, denoted Axis $(H)$. Moreover, if $h$ is a pseudo-Anosov homeomorphism representing $H$, with stable and unstable foliations $\mathcal{F}_{x}, \mathcal{F}_{y}$ and expansion factor $\lambda$, then $\operatorname{Axis}(H)=\gamma\left(\mathcal{F}_{x}, \mathcal{F}_{y}\right)$ and the translation length of $H$ equals $\log (\lambda)$.

Note that by a theorem of Bers [Ber78], a mapping class $H \in \mathcal{M}$ is pseudoAnosov if and only if the function $\sigma \mapsto d(H, H \sigma)$ has a positive minumum in $\mathcal{T}$; moreover this minimum is acheived precisely on $\operatorname{Axis}(H)$.

\subsection{Quasi-isometries}

Given $K \geq 1, C \geq 0$, a $(K, C)$ quasi-isometry between metric spaces is a map $f: X \rightarrow Y$ such that:

(1) For all $x_{1}, x_{2} \in X$ we have

$$
\frac{1}{K} d_{X}\left(x_{1}, x_{2}\right)-C \leq d_{Y}\left(f\left(x_{1}\right), f\left(x_{2}\right)\right) \leq K d_{X}\left(x_{1}, x_{2}\right)+C
$$


(2) $d_{Y}(y, f(X)) \leq C$ for each $y \in Y$.

If $f$ satisfies (1) but not necessarily (2) then it is called a $(K, C)$ quasi-isometric embedding. A quasi-isometric embedding $f: \mathbf{R} \rightarrow X$ is a quasigeodesic in $X$.

A coarse inverse of a quasi-isometry $f: X \rightarrow Y$ is a quasi-isometry $g: Y \rightarrow X$ such that, for some constant $C^{\prime}>0$, we have $d(g \circ f(x), x)<C^{\prime}$ and $d(f \circ g(y), y)<$ $C^{\prime}$ for all $x \in X$ and $y \in Y$. Every $(K, C)$ quasi-isometry $f: X \rightarrow Y$ has a $K, C^{\prime}$ coarse inverse $g: Y \rightarrow X$, where $C^{\prime}$ depends only on $K, C$ : for each $y \in Y$ define $g(y)$ to be any point $x \in X$ such that $d(f(x), y) \leq C$.

A fundamental fact observed by Efremovich, by Milnor [Mil68], and by Švarc, which we use repeatedly without mention, states that if a group $G$ acts properly discontinuously and cocompactly by isometries on a proper geodesic metric space $X$, then $G$ is finitely generated, and $X$ is quasi-isometric to $G$ equipped with the word metric.

Given a metric space $X$ and $A \subset X$, we denote $\operatorname{Nbhd}_{r}(A)=\{x \in X \mid d(x, r) \leq$ $A\}$, and given $A, B \subset X$, we denote the Hausdorff distance by

$$
d_{\mathcal{H}}(A, B)=\inf \left\{r \in[0, \infty] \mid A \subset \operatorname{Nbhd}_{r}(B) \text { and } B \subset \operatorname{Nbhd}_{r}(A)\right\}
$$

Given a metric space $X$, the self quasi-isometries of $X$ are denoted $\widehat{\mathrm{QI}}(X)$. Define the coarse equivalence relation on $\widehat{\mathrm{QI}}(X)$ where $f, g \in \widehat{\mathrm{QI}}(X)$ are coarsely equivalent, denoted $f \underset{c}{\approx} g$, if

$$
\sup _{x \in X} d(f x, g x)=C<\infty
$$

We call $C$ the coarseness constant. Composition of elements of $\widehat{\mathrm{QI}}(X)$ gives a well-defined binary operation on the set of coarse equivalence classes of self quasiisometries of $X$, defining a group $\mathrm{QI}(X)$, the quasi-isometry group of $X$. If $h: X \rightarrow$ $Y$ is a quasi-isometry of metric spaces then $h$ induces an isomorphism $\mathrm{QI}(X) \rightarrow$ $\mathrm{QI}(Y)$. In particular, when $\Gamma$ is a finitely generated group the identity map is a quasi-isometry with respect to the word metrics of any two finite generating sets, and so the quasi-isometry group $\mathrm{QI}(\Gamma)$ is independent of choice of word metric on $\Gamma$.

A quasi-action of a group $G$ on a metric space $X$ is a map $G \times X \rightarrow X$, denoted $(g, x) \mapsto g \cdot x$, such that for some $K \geq 1, C \geq 0$ we have:

- For each $g \in G$ the map $x \mapsto g \cdot x$ is a $(K, C)$-quasi-isometry.

- For each $g, h \in G, x \in X$ we have $d(g h \cdot x, g \cdot(h \cdot x)) \leq C$; in other words, $L_{g h} \underset{c}{\approx} L_{g} \circ L_{h}$ with coarseness constant independent of $g, h$, where $L_{\gamma}$ means left multiplication by $\gamma$. 
The quasi-action is cobounded if there exists a bounded subset $D$ having nonempty intersection with every orbit of the quasi-action. The quasi-action is proper if for each $R>0$ there exists an integer $m \geq 0$ such that for any $x, y \in X$ the cardinality of the set $\{g \in G \mid(g \cdot B(x, R)) \cap B(y, R) \neq \emptyset\}$ is at most $m$.

A fundamental principle of geometric group theory says that if a finitely generated group $G$ is quasi-isometric to a metric space $X$, then the left action of $G$ on itself, when conjugated by a quasi-isometry $G \rightarrow X$, defines a cobounded, proper quasi-action of $G$ on $X$. To be precise, if we have coarsely inverse quasi-isometries $h: X \rightarrow G, \bar{h}: G \rightarrow X$, then the formula $g \cdot x=\bar{h}(g h(x))$ defines a cobounded, proper quasi-action of $G$ on $X$.

\section{Schottky groups on Teichmüller space}

In this section we recall from [FM00b] the motivation for and definition of Schottky subgroups of mapping class groups; see that paper for details and proofs.

Recall that a Schottky group in $\operatorname{Isom}\left(\mathbf{H}^{n}\right)$ is a discrete, free use convex cocompact terminology subgroup $F$ such that every orbit is quasiconvex in $\operatorname{Isom}\left(\mathbf{H}^{n}\right)$. Equivalently, letting $\Lambda$ be the limit set of $F$ and $\mathcal{H} \Lambda$ the convex hull of $\Lambda$, the action of $F$ on $\mathcal{H} \Lambda$ is cocompact; it follows that $\mathcal{H} \Lambda$ is quasi-isometric to $F$, and this quasi-isometry extends continuously to an $F$-equivariant homeomorphism between $\Lambda$ and the Gromov boundary of $F$. This equivalence follows from the fact that $\mathbf{H}^{n}$ itself is a $\delta$-hyperbolic metric space, and in fact the same (or closely analogous) equivalence holds for free subgroups of word hyperbolic groups, thereby providing a theory of Schottky subgroups of word hyperbolic groups.

In [FM00b] we mimic this setup for free subgroups $F$ of $\operatorname{Isom}(\mathcal{T})=\mathcal{M}$. The tricky part is that Teichmüller space $\mathcal{T}$ is not $\delta$-hyperbolic. Nevertheless, when the properties above for Schottky subgroups of $\operatorname{Isom}\left(\mathbf{H}^{n}\right)$ are carefully translated into the language of $\operatorname{Isom}(\mathcal{T})$, the results of Minsky [Min96] provide enough negative curvature in $\mathcal{T}$ to prove the equivalence of various notions of Schottkiness for $F$. In addition, one of the main theorems of [FM00b] is that the Schottky condition characterizes precisely those free subgroups $F$ for which $\pi_{1}(\Sigma) \rtimes F$ is word hyperbolic.

Theorem 3.1 (Schottky groups: Definitions). Let $F$ be a finite rank free subgroup of $\mathcal{M}(\Sigma)$. The following are equivalent:

1. Orbit quasiconvexity Each orbit $\mathcal{O}$ of the action of $F$ on $\mathcal{T}$ is quasiconvex in $\mathcal{T}$, i.e. there is a constant $A$ such that for any $x, y \in \mathcal{O}$, the geodesic $\overline{x y}$ is contained in the A-neighborhood of $\mathcal{O}$. 
2. Weak convex cocompactness There is a continuous, F-equivariant embedding of the Gromov boundary $\partial F$ into $\mathbf{P} \mathcal{M} F$, with image denoted $\Lambda$, satisfying the following:

(1) For any $\xi \neq \eta \in \Lambda$ there is a geodesic $\overleftrightarrow{(\xi, \eta)}$ in $\mathcal{T}$; let

$$
\mathcal{H} \Lambda=\cup\{\overleftrightarrow{(\xi, \eta)} \mid \xi \neq \eta \in \Lambda\}
$$

be the weak convex hull of $\Lambda$, and let $\overline{\mathcal{H}} \Lambda=\mathcal{H} \Lambda \cup \Lambda$.

(2) The F-equivariant homeomorphism $\Lambda \rightarrow \partial F$ extends to an $F$-equivariant map

$$
(\overline{\mathcal{H}} \Lambda, \Lambda, \mathcal{H} \Lambda) \rightarrow(F \cup \partial F, \partial F, F)
$$

which is continuous at each point of $\Lambda$ and which restricts to a quasiisometry $\mathcal{H} \Lambda \rightarrow F$, with respect to the Teichmüller metric on $\mathcal{H} \Lambda$ and the word metric on $F$.

3. Word hyperbolic extension The extension group $\Gamma_{F}=\pi_{1}(\Sigma) \rtimes F$ is word hyperbolic.

Remark. It follows from the weak convex cocompactness property that for each geodesic $\overleftrightarrow{(\xi, \eta)}$ in $\mathcal{H} \Lambda$, the image of $\overleftrightarrow{(\xi, \eta)}$ in $F$ is a quasigeodesic whose ends converge in $F \cup \partial F$ to the images of $\xi, \eta$ respectively. It also follows that each nontrivial element $f \in F$ is pseudo-Anosov, because $f$ has an axis in the Cayley graph of $F$ and so $f$ has an axis in $\mathcal{T}$.

Theorem 3.1 is proved in [FM00b]. We call a subgroup $F$ satisfying any one of the equivalent conditions of Theorem 3.1 a Schottky subgroup of $\mathcal{M}(\Sigma)$, or a Schottky group of mapping classes. These groups exist in abundance:

Theorem 3.2 (Abundance of Schottky groups). Let $\phi_{1}, \ldots, \phi_{n}$ be a collection of $n$ independent pseudo-Anosov elements of $\mathcal{M}(\Sigma)$. Then for any sufficiently large natural numbers $a_{1}, \ldots, a_{n}$, the subgroup of $\mathcal{M}(\Sigma)$ generated by $\phi_{1}^{a_{1}}, \ldots, \phi_{n}^{a_{n}}$ is a Schottky subgroup.

Proof. As noted in [FM00b], this follows from Theorem 3.1 together with the main result of $[\operatorname{Mos} 97]$. 


\section{The geometry and topology of $\Gamma_{H}$}

\subsection{A geometric model for $\Gamma_{H}$}

Let $H$ be a Schottky subgroup of $\mathcal{M}=\operatorname{Isom}(\mathcal{T})$. We now build a contractible, piecewise-Riemannian 3-complex $X_{H}$ on which $\Gamma_{H}$ acts freely, properly discontinuously, and cocompactly by isometries, so that $\Gamma_{H}$ is quasi-isometric to $X$.

Choose a free generating set $h_{1}, \ldots, h_{n} \in \mathcal{M}$ for $H$. Let $\Delta$ be a graph with $n$ edges $\delta_{1}, \ldots, \delta_{n}$, each with one end at a common vertex $v_{0}$ of valence $n$, and with opposite ends at valence one vertices $v_{1}, \ldots, v_{n}$, respectively. Let $R$ be the rose with $n$ petals obtained from $\Delta$ by identifying $v_{i}$ with $v_{0}$ for each $i=1, \ldots, n$, and identify $\pi_{1}\left(R, v_{0}\right)$ with $H$ so that the homotopy class of the loop $\left[\delta_{i}\right]$ is identified with $h_{i}$.

On the product $\Sigma \times \Delta$ make the following identifications: for each $i$ choose a homeomorphism $\eta_{i}: \Sigma \rightarrow \Sigma$ representing $h_{i}$ and identify $\Sigma \times v_{n}$ with $\Sigma \times v_{0}$ by identifying $\left(x, v_{0}\right) \sim\left(\eta_{i}(x), v_{i}\right)$ for each $x \in \Sigma$. Let $K_{H}$ be the quotient 3-complex, and so we obtain a locally trivial fiber bundle $K_{H} \rightarrow R$ with fiber $\Sigma$ and with monodromy $H$. Up to a bundle isomorphism homotopic to the identity, $K_{H}$ does not depend on the choices of representatives $\eta_{i}$. By Van Kampen's Theorem we have an isomorphism $\pi_{1}\left(K_{H}\right) \approx \Gamma_{H}$.

Let $X_{H}$ be the universal cover of $K_{H}$. Hence $\Gamma_{H}$ acts properly discontinuously and cocompactly on $X_{H}$, with quotient $K_{H}$. We now specify a metric on $X_{H}$ for which this action is isometric.

Let $T_{H}$ be the universal cover of $R$, an infinite, regular, $2 n$-valent tree, regarded as the Cayley graph for $H$. The universal cover of $\Sigma$ is the Poincaré disc $D$. The bundle $K_{H} \rightarrow R$ with fiber $\Sigma$ lifts to a locally trivial fiber bundle $\pi: X_{H} \rightarrow T_{H}$ with fiber $D$, and it follows that $X_{H}$ is homeomorphic to $D \times T_{H}$. In order to construct a $\Gamma_{H}$-equivariant metric on $X_{H}$ the metrics on the fibers of $\pi$ must be appropriately "twisted" by considering the action of $H$ on $\mathcal{T}$.

We can embed the graph $\Delta$ in the tree $T_{H}$ as a fundamental domain for the action of $H$, so that the restriction of the universal covering map $T_{H} \rightarrow R$ agrees with the quotient map $\Delta \rightarrow R$. Pick a base point $\sigma_{0}$ in the Teichmüller space $\mathcal{T}$. Choose a map $\rho: \Delta \rightarrow \mathcal{T}$ taking $v_{0}$ to $\sigma_{0}$, taking $v_{i}$ to $h_{i}\left(\sigma_{0}\right)$, and taking $\delta_{i}$ to a smooth path between $\sigma_{0}$ and $h_{i}\left(\sigma_{0}\right)$, say the Teichmüller geodesic. Extend $H$-equivariantly to obtain a map $\rho: T_{H} \rightarrow \mathcal{T}$. Henceforth we shall fix the map $\rho$, and $\operatorname{arcs}$ in the tree $T_{H}$ will be parameterized by arc length in $\mathcal{T}$ with respect to the map $\rho$. We use $\tau$ as a variable taking values in $T_{H}$ and $d \tau$ as the arc length parameter in $T_{H}$.

Given an $\operatorname{arc} \alpha \subset T_{H}$, we can impose a hyperbolic structure on leaves of $\Sigma \times \alpha$ in the form of a Riemannian metric $g_{\tau}$ on each $\Sigma \times \tau$, so that the conformal class of $g_{\tau}$ 
represents the point $\tau \in \mathcal{T}$, and so that the metrics $g_{\tau}$ vary smoothly with $\tau \in \alpha$. This can be obtained for instance by choosing a pair-of-pants decomposition of $\Sigma$ and using the associated Fenchel-Nielsen coordinates for $\mathcal{T}$ (see [FM00b] for a further discussion). Then we may extend to obtain a piecewise smooth Riemannian metric on $\Sigma \times \alpha$ by the formula

$$
d s^{2}=g_{\tau}^{2}+d \tau^{2}
$$

This metric is smooth on $\Sigma \times \alpha$ except over the vertices of $T_{H}$ in $\alpha$.

Applying this to each edge $\delta_{i}$ of $\Delta$ we obtain hyperbolic structures on the fibers of $\Sigma \times \Delta$, varying smoothly over each edge $\delta_{i}$, so that for each $\tau \in \Delta$ the hyperbolic surface $\Sigma \times \tau$ represents $\rho(\tau) \in \mathcal{T}$. We therefore obtain a piecewise smooth Riemannian metric on the 3-complex $\Sigma \times \Delta$. Since $h_{i}\left(v_{0}\right)=v_{i}$ it follows that there is a unique isometry $\eta_{i}: \Sigma \times v_{0} \rightarrow \Sigma \times v_{i}$ representing the mapping class $h_{i}$, and we use these choices of $\eta_{i}$ to construct $K_{H}$ (here, for each $\tau \in \Delta$, we are implicitly identifying $\Sigma \times \tau$ with $\Sigma$ by projection to the first factor). Thus we have defined a piecewise smooth Riemannian metric on $K_{H}$ whose restriction to each fiber of the bundle $K_{H} \rightarrow R$ is a hyperbolic metric on that fiber.

Lifting the metric on $K_{H}$ we obtain a piecewise Riemannian metric on the universal cover $X_{H}$, whose restrictions to the fibers of the bundle $\pi: X_{H} \rightarrow T_{H}$ give a continuously varying family of hyperbolic metrics $\bar{g}_{\tau}$ on the fibers, parameterized by $\tau \in T_{H}$. Each fiber is isometric to $\mathbf{H}^{2}$. For each arc $\alpha$ of $T_{H}$ the sub-bundle $\pi^{-1}(\alpha)$ has the form $D \times \alpha$ and the Riemannian metric has the form

$$
d \bar{s}^{2}=\bar{g}_{\tau}^{2}+d \tau^{2}
$$

where $\bar{g}_{\tau}$ is the lift to $D \times \tau$ of the metric $g_{\tau}$ on the appropriate fiber of $K_{H}$.

With respect to the piecewise Riemannian metric and the associated geodesic metric on $X_{H}$, the group $\Gamma_{H}$ acts properly discontinuously and cocompactly by isometries.

\subsection{What it means to coarsely respect a pattern}

A pattern in a metric space is simply a collection of subsets. We will loosely use the term foliation to refer to a pattern forming a partition of the space into disjoint subsets called leaves, and in that context the pattern itself will be called the leaf space.

Let $X, Y$ be metric spaces, and let $\mathcal{F}, \mathcal{G}$ be patterns in $X, Y$ respectively. A quasi-isometry $\phi: X \rightarrow Y$ is said to coarsely respect the patterns $\mathcal{F}, \mathcal{G}$ if there exists a number $A \geq 0$ and a map $h: \mathcal{F} \rightarrow \mathcal{G}$ such that for each element $L \in \mathcal{F}$ we have

$$
d_{\mathcal{H}}(\phi(L), h(L)) \leq A
$$


and if further a similar statement holds for a coarse inverse of $\phi$. When distinct elements of $\mathcal{F}$ have infinite Hausdorff distance in $X$, and similarly for $\mathcal{G}$, then $h$ is a bijection and it is uniquely determined by the quasi-isometry $\phi$.

An isometry of $X_{H}$ respects many different patterns in $X_{H}$. The idea of the proof of Theorem 1.3 is to show that an arbitrary quasi-isometry $f$ of $\Gamma_{H}$ (hence of $X_{H}$ ) preserves more and more structure, in the sense of coarsely respecting finer and finer patterns, until so much structure is preserved that $f$ must actually be a bounded distance from an element of the extension group $\Gamma_{\mathcal{C}}$ defined in Theorem 1.3.

\subsection{Hyperplanes and the horizontal foliation}

The bundle $\pi: X_{H} \rightarrow T_{H}$ associated to the group $\Gamma_{H}$ determines two important patterns in $X_{H}$ : the horizontal foliation and the pattern of hyperplanes.

The horizontal foliation of $X_{H}$ is the pattern of fibers of $\pi$, subsets $D_{\tau}=$ $\pi^{-1}(\tau), \tau \in T_{H}$, each called a horizontal leaf of $X_{H}$. The leaf space of the horizontal foliation is identified with $T_{H}$ via the bundle map $\pi$. Any two horizontal leaves have finite Hausdorff distance in $X_{H}$, in fact from the form of the metric on $X_{H}$ we see easily that $d_{\mathcal{H}}\left(D_{\tau}, D_{u}\right)=d(\tau, u)$ for all $\tau, u \in T_{H}$. In other words, the bundle map $\pi$ induces an isometry between the horizontal foliation equipped with the Hausdorff metric and the metric tree $T_{H}$. As noted earlier, the metric on $X_{H}$ restricts to a metric $\bar{g}_{\tau}$ on each horizontal leaf $D_{\tau}$ making $D_{\tau}$ isometric to $\mathbf{H}^{2}$.

A hyperplane in $X_{H}$ is any set $P_{w}=\pi^{-1}(w)$, where $w$ is a bi-infinite geodesic in $T_{H}$. The metric on $X_{H}$ restricts to a piecewise Riemannian metric on $P_{w}$ of the form $\tilde{g}_{\tau}^{2}+d \tau^{2}$, with respect to the coordinates $P_{w} \approx D \times w$. Any two distinct hyperplanes in $X_{H}$ have infinite Hausdorff distance.

The pattern of hyperplanes in $X_{H}$ is the first pattern which must be coarsely respected by a quasi-isometry.

Proposition 4.1 (Hyperplanes respected). Any quasi-isometry $f: X_{H} \rightarrow X_{H}$ coarsely respects the pattern of hyperplanes in $X_{H}$, with coarseness constant depending only on the quasi-isometry constants of $f$.

In other words, $f$ must map each hyperplane in $X_{H}$ a uniform Hausdorff distance from some other hyperplane in $X_{H}$. Since distinct hyperplanes have infinite Hausdorff distance in $X_{H}$ it follows that $f$ induces a bijection on the pattern of hyperplanes; when $f$ is understood we denote this bijection $P_{w} \mapsto P_{w^{\prime}}$.

Each hyperplane $P_{w}$ is uniformly properly embedded in $X_{H}$, that is, there is a proper function $r:[0, \infty) \rightarrow[0, \infty)$ independent of $w$ such that for all $x, y \in P_{w}$ we have:

$$
d_{X_{H}}(x, y) \geq r\left(d_{P_{w}}(x, y)\right)
$$


By applying Proposition 4.1 together with a simple general principle (made explicit in Lemma 2.1 of [FM00a]) we have for any quasi-isometry $f: X_{H} \rightarrow X_{H}$ the following fact: for each hyperplane $P_{w}$, the map $f$ induces via restriction composed with nearest-point projection, a quasi-isometry $\phi: P_{w} \rightarrow P_{w^{\prime}}$; the quasi-isometry constants for $\phi$ depend only on those for $f$.

The horizontal foliation on $X_{H}$ restricts to a horizontal foliation on $P_{w}$. Using the coordinates $P_{w} \approx D \times \mathbf{R}$ as described above, the horizontal leaves in $P_{w}$ are of the form $D_{t}=D \times t, t \in \mathbf{R}$. The leaf space of this foliation is $\mathbf{R}$ and we have $d_{\mathcal{H}}\left(D_{s}, D_{t}\right)=|s-t|$ using Hausdorff distance in $P_{w}$.

The fact that $X_{H}$ fibers over a tree $T_{H}$ with nontrivial branching restricts the behavior of $\phi$ as follows:

Proposition 4.2 (Horizontal foliation respected). For any quasi-isometry $f: X_{H} \rightarrow$ $X_{H}$ and any hyperplane $P_{w}$, the induced quasi-isometry $\phi: P_{w} \rightarrow P_{w^{\prime}}$ uniformly coarsely respects horizontal foliations.

In other words, there exists a constant $A$ independent of $w$ such that for any horizontal leaf $D_{t} \subset P_{w}$ there is a horizontal leaf $D_{t^{\prime}} \subset P_{w^{\prime}}$ with $d_{\mathcal{H}}\left(\phi\left(D_{t}\right), D_{t^{\prime}}\right) \leq$ A

This is precisely where the assumption is used that $H$ has rank greater than one.

Proof of Propositions 4.1 and 4.2. Since $H$ has rank greater than one, its Cayley graph $T_{H}$ is a bushy tree, meaning that each point of $T_{H}$ is within some fixed distance $\beta=1$ of some vertex $v$ such that $T-v$ has at least 3 unbounded components. We can thus apply the following result, which is Theorem 7.7 of [FM00a], to the metric fibration $\pi: X_{H} \rightarrow T_{H}$.

Lemma 4.3. Let $\pi: X \rightarrow T, \pi^{\prime}: X^{\prime} \rightarrow T^{\prime}$ be metric fibrations over bushy trees $T, T^{\prime}$, such that the fibers of $\pi$ and $\pi^{\prime}$ are contractible $n$-manifolds for some $n$. Let $f: X \rightarrow X^{\prime}$ be a quasi-isometry. Then there exists a constant $A$, depending only on the metric fibration data of $\pi, \pi^{\prime}$, the quasi-isometry data for $f$, and the constant $\beta$, such that:

(1) For each hyperplane $P \subset X$ there exists a unique hyperplane $Q \subset X^{\prime}$ such that $d_{\mathcal{H}}(f(P), Q) \leq A$.

(2) For each horizontal leaf $L \subset X$ there is a horizontal leaf $L^{\prime} \subset X^{\prime}$ such that $d_{\mathcal{H}}\left(f(L), L^{\prime}\right) \leq A$.

Proposition 4.1 is an immediate consequence. To obtain Proposition 4.2, consider a horizontal leaf $D_{t}$ of $P_{w}$. By applying Lemma 4.3 we obtain a horizontal 
leaf $D_{s}$ of $X_{H}$ uniformly Hausdorff close to $f\left(D_{t}\right)$. But $f\left(P_{w}\right)$ is uniformly Hausdorff close to $P_{w^{\prime}}$ and so $D_{s}$ is uniformly Hausdorff close to some horizontal leaf $D_{t^{\prime}} \subset P_{w^{\prime}}$. Finally, the closest point projection $f\left(P_{w}\right) \mapsto P_{w^{\prime}}$ moves points a uniformly bounded distance, and so $f\left(P_{w}\right)$ is a uniformly Hausdorff close to its closest point projection in $P_{w^{\prime}}$.

\subsection{The singular solv metric on a hyperplane}

In this subsection we find a different metric on hyperplanes $P_{w}$ that will allow us to apply ideas from pseudo-Anosov dynamics.

Associated to each geodesic $\gamma$ in $\mathcal{T}$ we recall the construction of a "singular SOLV metric" on $D \times \gamma$. Choose a normalized transverse pair of measured foliations $\mathcal{F}_{x}, \mathcal{F}_{y}$ on $\Sigma$, with transverse measures $|d y|,|d x|$ respectively, so that $\gamma=\gamma\left(\mathcal{F}_{x}, \mathcal{F}_{y}\right)$; we can parameterize $\gamma$ by arc length:

$$
\gamma(t)=\sigma\left(e^{t} \mathcal{F}_{x}, e^{-t} \mathcal{F}_{y}\right)
$$

For each $t$ we have a singular Euclidean metric $\mu_{t}=\mu\left(e^{t} \mathcal{F}_{x}, e^{-t} \mathcal{F}_{y}\right)$, that is,

$$
d \mu_{t}^{2}=e^{-2 t} d x^{2}+e^{2 t} d y^{2}
$$

Note that the transverse pair $\left(e^{t} \mathcal{F}_{x}, e^{-t} \mathcal{F}_{y}\right)$ is normalized for all $t$, that is, Area $\left(\mu_{t}\right)=$ $\int_{\Sigma} e^{t}|d y| e^{-t}|d x|=\int_{\Sigma}|d x||d y|=1$. Lifting to the universal cover $D=\widetilde{\Sigma}$ we obtain a transverse pair of measured foliations $\widetilde{\mathcal{F}}_{x}, \widetilde{\mathcal{F}}_{y}$ with transverse measures still denoted $|d y|,|d x|$, and a one-parameter family of singular Euclidean metrics $\tilde{\mu}_{t}$ on $D$, given by

$$
d \tilde{\mu}_{t}^{2}=e^{-2 t} d x^{2}+e^{2 t} d y^{2}
$$

Now we can define a singular Riemannian metric on $D \times \gamma$ by the formula

$$
\begin{aligned}
d s^{2} & =d \tilde{\mu}_{t}^{2}+d t^{2} \\
& =e^{-2 t} d x^{2}+e^{2 t} d y^{2}+d t^{2}
\end{aligned}
$$

The singular locus of this metric is a family of vertical lines in $D \times \gamma$, whose intersection with the fiber $D \times t$ is precisely the singular set of $e^{t} \widetilde{\mathcal{F}}_{x}, e^{-t} \widetilde{\mathcal{F}}_{y}$. The Riemannian metric extends across the singular locus to a complete geodesic metric on $D \times \gamma$. The space $D \times \gamma$ with this metric is called the singular SOLV space associated to $\gamma$, denoted $Q_{\gamma}$. The reason for the terminology is that away from the singular locus the metric is locally modelled on 3-dimensional SOLV-geometry, and at the singular locus (each component of which is a line) the metric is modelled on some number of "half soLv's" glued together. 
Note that the singular SOLV-metric on $Q_{\gamma}$ is uniquely determined by the fibration $Q_{\gamma} \rightarrow \gamma$ together with the family of $x y$-structures $e^{t} \widetilde{\mathcal{F}}_{x}, e^{-t} \widetilde{\mathcal{F}}_{y}$ : in each leaf; the measured foliation $e^{t} \widetilde{\mathcal{F}}_{x}$ provides the $e^{2 t} d y$ term and the measured foliation $e^{-t} \widetilde{\mathcal{F}}_{y}$ provides the $e^{-2 t} d x$ term, and the map to $\gamma$ with the arc length parameter $t$ provides the $d t^{2}$ term.

In summary, we have a bijective correspondence

$\{$ geodesics $\gamma$ in $\mathcal{T}\} \leftrightarrow\left\{\pi_{1}(\Sigma)\right.$-equivariant singular SOLV metrics on $\left.D \times \mathbf{R}\right\}$

$x y$-structures. For convenience we shall define an $x y$-structure on a surface $S$ to be a transverse pair of measured foliations $\mathcal{F}_{x}, \mathcal{F}_{y}$. We will work with $x y$-structures on $\Sigma$ as well as the lifted structures on the universal cover $D$.

An affine automorphism of an $x y$-structure on $S$ is a homeomorphism of $S$ which respects the (unordered) pair of (unmeasured) foliations $\left\{\mathcal{F}_{x}, \mathcal{F}_{y}\right\}$, multiplying the transverse measure on one by $\lambda>0$ and multiplying the transverse measure on the other by $1 / \lambda$. Let $\operatorname{Aff}\left(\mathcal{F}_{x}, \mathcal{F}_{y}\right)$ be the group of affine automorphisms of the $x y$-structure $\mathcal{F}_{x}, \mathcal{F}_{y}$. There is a subgroup of index $\leq 2 \operatorname{in} \operatorname{Aff}\left(\mathcal{F}_{x}, \mathcal{F}_{y}\right)$, denoted $\operatorname{Aff}_{+}\left(\mathcal{F}_{x}, \mathcal{F}_{y}\right)$, which respects the ordered pair of (unmeasured) measured foliations $\left(\mathcal{F}_{x}, \mathcal{F}_{y}\right)$, with transverse measures multiplied as described above. There is a homomorphism

$$
\text { Stretch: } \operatorname{Aff}\left(\mathcal{F}_{x}, \mathcal{F}_{y}\right) \rightarrow \operatorname{SL}(2, \mathbf{R})
$$

whose image lies in the subgroup of $\mathrm{SL}(2, \mathbf{R})$ corresponding to matrices which are either zero off the diagonal or zero on the diagonal, as follows. Given $\phi \in$ $\operatorname{Aff}\left(\mathcal{F}_{x}, \mathcal{F}_{y}\right)$ : if $\phi\left(\mathcal{F}_{x}\right)=\lambda \mathcal{F}_{x}$ and $\phi\left(\mathcal{F}_{y}\right)=\frac{1}{\lambda} \mathcal{F}_{y}$ then $\operatorname{Stretch}(\phi)$ is the matrix $\left(\begin{array}{cc}\lambda & 0 \\ 0 & 1 / \lambda\end{array}\right)$; whereas if $\phi\left(\mathcal{F}_{x}\right)=\lambda \mathcal{F}_{y}$ and $\phi\left(\mathcal{F}_{y}\right)=\frac{1}{\lambda} \mathcal{F}_{x}$ then $\operatorname{Stretch}(\phi)$ is the matrix $\left(\begin{array}{cc}0 & \lambda \\ 1 / \lambda & 0\end{array}\right)$. In the cases of interest where $S=\Sigma$ or $D$, the image of the homomorphism, denoted $\operatorname{Stretch}\left(\mathcal{F}_{x}, \mathcal{F}_{y}\right)$, is a discrete $\operatorname{subgroup~of~} \operatorname{SL}(2, \mathbf{R})$; discreteness follows easily using the fact that the singular set of the pair $\mathcal{F}_{x}, \mathcal{F}_{y}$ forms a nonempty, discrete net in $S$. As a consequence, $\operatorname{Stretch}\left(\mathcal{F}_{x}, \mathcal{F}_{y}\right)$ is isomorphic to either $D_{\infty}, \mathbf{Z}, \mathbf{Z} / 2$, or the trivial group. The kernel of the stretch homomorphism is the group $\operatorname{Isom}_{+}\left(\mathcal{F}_{x}, \mathcal{F}_{y}\right)$ of automorphisms of the ordered pair of measured foliations $\mathcal{F}_{x}, \mathcal{F}_{y}$.

We obtain a short exact sequence

$$
1 \rightarrow \operatorname{Isom}_{+}\left(\mathcal{F}_{x}, \mathcal{F}_{y}\right) \rightarrow \operatorname{Aff}\left(\mathcal{F}_{x}, \mathcal{F}_{y}\right) \rightarrow \operatorname{Stretch}\left(\mathcal{F}_{x}, \mathcal{F}_{y}\right) \rightarrow 1
$$

where $\operatorname{Isom}_{+}\left(\mathcal{F}_{x}, \mathcal{F}_{y}\right)$ is the group of automorphisms of the ordered pair of measured foliations $\mathcal{F}_{x}, \mathcal{F}_{y}$.

Given a geodesic $\gamma=\gamma\left(\mathcal{F}_{x}, \mathcal{F}_{y}\right)$ in $\mathcal{T}$, the universal cover $D=\widetilde{\Sigma}$ with the lifted $x y$-structure $\widetilde{\mathcal{F}}_{x}, \widetilde{\mathcal{F}}_{y}$ may be identified with a certain horizontal fiber of $Q_{\gamma}$. 
With respect to this identification, every isometry of the singular SOLV-manifold $Q_{\gamma}$ respects the horizontal foliation, and vertical projection of an isometry onto $D$ defines an $x y$-affine automorphism of $\widetilde{\mathcal{F}}_{x}, \widetilde{\mathcal{F}}_{y}$. Every $x y$-affine automorphism arises in this manner, leading to an isomorphism between $\operatorname{Isom}\left(Q_{\gamma}\right)$ and $\operatorname{Aff}\left(\widetilde{\mathcal{F}}_{x}, \widetilde{\mathcal{F}}_{y}\right)$. This leads in turn to an isomorphism of short exact sequences as follows:

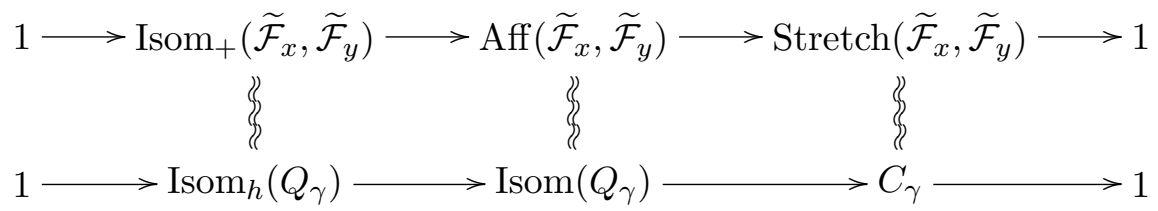

where $\operatorname{Isom}_{h}\left(Q_{\gamma}\right)$ is the subgroup of $\operatorname{Isom}\left(Q_{\gamma}\right)$ preserving each horizontal leaf, and $C_{\gamma}=\operatorname{Isom}\left(Q_{\gamma}\right) / \operatorname{Isom}_{h}\left(Q_{\gamma}\right)$; the latter group is isomorphic to $D_{\infty}, \mathbf{Z}, \mathbf{Z} / 2$, or the trivial group.

We also have an isomorphism of short exact sequences

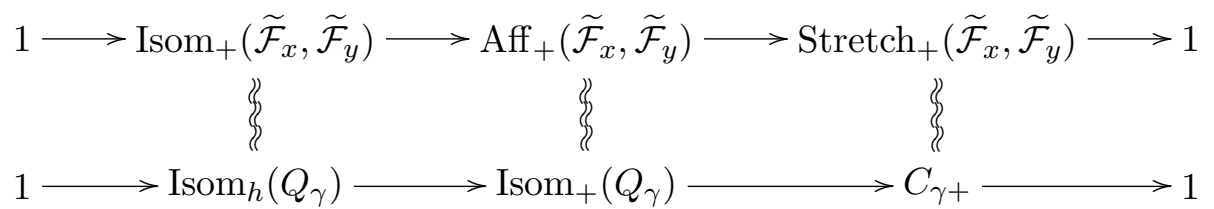

where $\operatorname{Aff}_{+}\left(\widetilde{\mathcal{F}}_{x}, \widetilde{\mathcal{F}}_{y}\right)$ was defined earlier, $\operatorname{Stretch}_{+}\left(\widetilde{\mathcal{F}}_{x}, \widetilde{\mathcal{F}}_{y}\right)$ is the intersection of $\operatorname{Stretch}\left(\widetilde{\mathcal{F}}_{x}, \widetilde{\mathcal{F}}_{y}\right) \subset \operatorname{SL}(2, \mathbf{R})$ with the diagonal subgroup of $\operatorname{SL}(2, \mathbf{R}), \operatorname{Isom}_{+}\left(Q_{\gamma}\right)$ is the subgroup of $\operatorname{Isom}\left(Q_{\gamma}\right)$ preserving the transverse orientation on the horizontal foliation, and $C_{\gamma+}=\operatorname{Isom}_{+}\left(Q_{\gamma}\right) / \operatorname{Isom}_{h}\left(Q_{\gamma}\right)$; in each of these four cases, the + subscript induces a subgroup of index $\leq 2$. The group $\operatorname{Stretch}_{+}\left(\widetilde{\mathcal{F}}_{x}, \widetilde{\mathcal{F}}_{y}\right) \approx C_{\gamma+}$ is either $\mathbf{Z}$ or trivial.

Note that $C_{\gamma}$ contains the group $\operatorname{Stab}_{\mathcal{T}}(\gamma)=\{\Phi \in \operatorname{Isom}(\mathcal{T}) \mid \Phi(\gamma)=\gamma\}$, and we shall show in Lemma 6.2 that this containment has finite index.

Schottky groups and singular solv-manifolds. Now consider a Schottky group $H \subset \mathcal{M}$ with limit set $\Lambda \subset \mathbf{P} \mathcal{M} F$, and weak convex hull $\mathcal{H} \Lambda$. We have the Cayley graph $T_{H}$ and an $H$-equivariant immersion $\rho: T_{H} \rightarrow \mathcal{T}$; let $\bar{T}_{H}=T_{H} \cup \Lambda$. An immediate corollary of Theorem 3.1 is that $T_{H}$ and $\mathcal{H} \Lambda$ have finite Hausdorff distance in $\mathcal{T}$, and so we obtain an $H$-equivariant quasi-isometry $\theta: \mathcal{H} \Lambda \rightarrow T_{H}$ which moves points a uniformly bounded distance in $\mathcal{T}$. It also follows from Theorem 3.1 that the map $\theta$ extends, via the identity map on $\Lambda$, to an $H$-equivariant map $\bar{\theta}: \overline{\mathcal{H}} \Lambda \rightarrow \bar{T}_{H}$ which is continuous at each point of $\Lambda$. It follows immediately that for any $\xi \neq \eta \in \Lambda$, the restriction of $\theta$ to the geodesic $\overleftrightarrow{(\xi, \eta)}$ under $\theta$ is a 
quasigeodesic, with quasigeodesic constants independent of $\xi, \eta$, and the ends of this quasigeodesic converge in $\bar{T}_{H}$ to $\xi, \eta$ respectively; the unique geodesic in $T_{H}$ with these endpoints is denoted $\overline{\xi \eta}$. To summarize:

- The correspondence between geodesics $\gamma=\overleftrightarrow{(\xi, \eta)}$ in $\mathcal{H} \Lambda$ and geodesics $w=$ $\overline{\xi \eta}$ in $T_{H}$ is a bijection. We denote this correspondence by $w=w_{\gamma}, \gamma=\gamma_{w}$. Corresponding geodesics $\gamma, w_{\gamma}$ are uniformly Hausdorff close in $\mathcal{T}$.

We need to be a bit more precise. By lifting each geodesic in $\mathcal{H} \Lambda$ to the unit tangent bundle $T^{1} \mathcal{T}$ we get a closed subset of $T^{1} \mathcal{T}$ invariant under the geodesic flow denoted $T \mathcal{H} \Lambda$. The space $T \mathcal{H} \Lambda$ is locally homeomorphic to $\Lambda \times \Lambda \times \mathbf{R}$, i.e. it is locally a Cantor set crossed with the line. We will often confuse a geodesic $\gamma$ in $\mathcal{H} \Lambda$ with its lift to $T \mathcal{H} \Lambda$; these geodesics form a lamination of $T \mathcal{H} \Lambda$. As we have said, the map $\theta: \mathcal{H} \Lambda \rightarrow T_{H}$ lifts to a map from $T \mathcal{H} \Lambda$ to $T_{H}$, taking each geodesic $\gamma$ to a quasigeodesic in $T_{H}$ uniformly Hausdorff close to $w_{\gamma}$. But then, by moving the map $T \mathcal{H} \Lambda \rightarrow T_{H}$ a bounded amount, we obtain a continuous map $\Theta: T \mathcal{H} \Lambda \rightarrow \mathcal{T}_{H}$ with the property that $\Theta(\gamma)=w_{\gamma}$ for each $\gamma$. The restriction to $\gamma$ is denoted $\Theta_{\gamma}: \gamma \rightarrow w_{\gamma}$, and this is a quasi-isometry with constants independent of $\gamma$, and we may take $\Theta_{\gamma}$ to be a homeomorphism.

Proposition 4.4 (Comparison of metrics). Given corresponding geodesics $w$ in $T_{H}$ and $\gamma=\gamma_{w}$ in $T \mathcal{H} \Lambda$, there exists a $\pi_{1} \Sigma$-equivariant, horizontal respecting quasi-isometry $F_{w}: P_{w} \rightarrow Q_{\gamma}$, with quasi-isometry constants independent of $w$, such that $F_{w}$ is a lift of the map $\Theta_{\gamma}^{-1}: w \rightarrow \gamma$.

In other words, the natural metric on each hyperplane of the geometric model space $X_{H}$ is uniformly quasi-isometric to a singular SOLV 3-manifold. This is the key place where we use the fact that $H$ is a Schottky group, and not just any free group of pseudo-Anosovs.

Proof. Recall that we have a $\Gamma_{H}$-equivariant $D$-bundle $X_{H} \rightarrow T_{H}$, carrying a $\Gamma_{H}$-equivariant piecewise Riemannian metric whose restriction to each fiber is isometric to $\mathbf{H}^{2}$, with $\Gamma_{H}$ acting cocompactly. Each of the spaces $P_{w}$ is embedded in $X_{H}$ as the inverse image of $w \subset T_{H}$.

We now define a $\Gamma_{H}$-equivariant $D$-bundle $\Xi \rightarrow T \mathcal{H} \Lambda$ in which each of the singular sOLV-manifolds $Q_{\gamma}$ sits, as follows.

First, note that each point of $T^{1} \mathcal{T}$ corresponds to (the isotopy class of) a normalized $x y$-structure $\left(\mathcal{F}_{x}, \mathcal{F}_{y}\right)$ on $\Sigma$. We may assemble these structures into an $\mathcal{M}$-equivariant $\Sigma$-bundle over $T^{1} \mathcal{T}$, each fiber equipped with an $x y$-structure in the appropriate isotopy class, so that the $x y$-strucures vary continuously as the base point in $T^{1} \mathcal{T}$ varies. Restricting to $T \mathcal{H} \Lambda$ we obtain an $H$-equivariant $x y$ - $\Sigma$-bundle $\Upsilon \rightarrow T \mathcal{H} \Lambda$. 
The universal cover of the $\Sigma$-bundle over $T^{1} \mathcal{T}$ is a $D$-bundle over $\mathcal{T}$, with smoothly varying $x y$-structures on the fibers, on which the $\pi_{1} \Sigma$ extension of $\mathcal{M}$ acts, namely the once-punctured mapping class group $\mathcal{M}(\Sigma, p)$. Restricting to $T \mathcal{H} \Lambda$ we obtain a $\Gamma_{H}$-equivariant $x y$-D-bundle $\Xi \rightarrow T \mathcal{H} \Lambda$. Restricting to any geodesic $\gamma \subset T \mathcal{H} \Lambda$ we obtain the fibration $Q_{\gamma} \rightarrow \gamma$. Note that the foliation of $T \mathcal{H} \Lambda$ by geodesics lifts to a foliation of $\Xi$ by 3 -manifolds: the 3 -manifold over the geodesic $\gamma \subset T \mathcal{H} \Lambda$ is $Q_{\gamma}$. The fiberwise $x y$-structures vary continuously in $\Xi$, and the arc length parameter on geodesics of $T \mathcal{H} \Lambda$ varies continuously; as noted earlier, these data determine singular SOLV-metrics on each $Q_{\gamma}$, and these metrics vary continuously in $\Xi$.

Now lift the $H$-equivariant map $\Theta: T \mathcal{H} \Lambda \rightarrow T_{H}$ to a $\Gamma_{H}$-equivariant continuous map of $D$-bundles, $\widetilde{\Theta}: \Xi \rightarrow X_{H}$, taking each fiber of $\Xi$ homeomorphically to the corresponding fiber of $X_{H}$, and taking each singular SOLV manifold $Q_{\gamma}$ to the corresponding singular Riemannian manifold $P_{w_{\gamma}}$.

By cocompactness of the $\Gamma_{H}$ actions on $\Xi$ and $X_{H}$, and by continuity of the $x y$-structures on fibers of $\Xi$ and the $\mathbf{H}^{2}$ structures on fibers of $X_{H}$, it follows that $\widetilde{\Theta}$ induces quasi-isometries from $\Xi$ fibers to $X_{H}$ fibers with uniform quasi-isometry constants; in particular, we get uniform quasi-isometries from the horizontal sets of $Q_{\gamma}$ to the horizontal sets of $P_{w_{\gamma}}$, over all geodesics $\gamma$ in $T \mathcal{H} \Lambda$. Moreover, since the map $\Theta: T \mathcal{H} \Lambda \rightarrow T_{H}$ is uniformly quasi-isometric from a geodesic $\gamma$ in $T \mathcal{H} \Lambda$ to the corresponding geodesic $w_{\gamma}$ in $T_{H}$, it follows that $\widetilde{\Theta}$ induces a uniform quasiisometry from the $|d t|$ term in the metric on $Q_{\gamma}$ to the $d \tau$ term in the metric on $P_{w_{\gamma}}$. This implies that the family of maps $Q_{\gamma} \rightarrow P_{w_{\gamma}}$ is uniformly quasi-isometric. $\diamond$

\section{Quasi-isometries remember the dynamics}

Let $H \subset \mathcal{M}$ be a Schottky subgroup and let $\phi: \Gamma_{H} \rightarrow \Gamma_{H}$ be a quasi-isometry. By Propositions 4.1, 4.2, and 4.4, to each bi-infinite path $w \in T_{H}$ there corresponds a bi-infinite path $w^{\prime} \in T_{H}^{\prime}$, and a horizontal respecting quasi-isometry $Q_{\gamma_{w}} \rightarrow Q_{\gamma_{w^{\prime}}}$ of singular SOLV spaces. In this section we study such quasi-isometries, and show that they must coarsely respect certain dynamically defined foliations.

\subsection{The vertical flow on $Q_{\gamma}$}

Let $\gamma$ be a fixed Teichmüller geodesic in $\mathcal{T}(\Sigma)$, and let $Q_{\gamma}$ be the associated singular SOLV metric on $D \times \gamma \approx D \times \mathbf{R}$. There is a natural flow on $Q_{\gamma}$ given by

$$
\Psi_{s}(x, t)=(x, t+s)
$$

This flow is called the vertical flow, and its orbits are called vertical geodesics or vertical flow lines in $Q_{\gamma}$. This flow is a pseudo-Anosov flow in the sense of 
[FM00c]. The stable and unstable foliations of $\Psi$ expand and contract at the uniform rate $e^{t}$. Singular orbits of $\Psi$ are the same as singular vertical geodesics of $Q_{\gamma}$.

There are three naturally defined foliations of $Q_{\gamma}$ which are invariant under the vertical flow: the codimension- 2 foliation by vertical flow lines; the codimension-1 weak stable foliation; and the codimension- 1 weak unstable foliation. Given a vertical flow line $\ell$, the weak stable (resp. unstable) leaf containing $\ell$ is the union of vertical flow lines that asymptote to $\ell$ as $t \rightarrow \infty$ (resp. $t \rightarrow-\infty$ ). Each of the weak stable and unstable foliations has some nonmanifold leaves, one such leaf containing each singular vertical geodesic. Note that for each leaf $L$ of the weak stable or unstable foliation, if $L$ is nonsingular then $L$ is isometric to a hyperbolic plane, whereas if $L$ is the singular leaf through a singular vertical geodesic $\ell$ then $L$ is isometric to a union of hyperbolic half-planes meeting along their common boundary $\ell$; in either case, $L$ can be expressed as a finite union of hyperbolic planes.

Our goal now is to use pseudo-Anosov dynamics to show that a horizontalrespecting quasi-isometry between two hyperplanes must also coarsely respect all of these foliations. In short: the quasi-isometry remembers the dynamics.

\subsection{Coarse intersection}

We need a basic notion from coarse topology.

Definition (Coarse intersection). A subset $W$ of a metric space $X$ is a coarse intersection of subsets $U, V \subset X$, denoted $W=U_{c} V$, if there exists $C_{0}$ such that for every $C \geq C_{0}$ there exists $A=A(C) \geq 0$ so that

$$
d_{\mathcal{H}}\left(\operatorname{Nbhd}_{C}(U) \cap \operatorname{Nbhd}_{C}(V), W\right) \leq A
$$

Note that although such a set $W$ may not exist, when it does exist then any two such sets are a bounded Hausdorff distance from each other. The function $A(C), C \geq C_{0}$ is called the coarse intersection function.

We will need the following fact, which is an elementary consequence of the definitions.

Lemma 5.1. For any quasi-isometry $f: X \rightarrow Y$ of metric spaces, and $U, V \subset X$, if $U \cap_{c} V$ exists then $f\left(U \cap_{c} V\right)$ is a coarse intersection of $f(U), f(V)$, with coarse intersection function depending only on the quasi-isometry constants for $f$ and the coarse intersection function for $U$ and $V$. 


\subsection{The dynamically defined foliations are respected}

In this subsection we prove the key proposition:

Proposition 5.2 (Stable and unstable foliations respected). Let $\gamma, \gamma^{\prime}$ be cobounded Teichmüller geodesics. Then any horizontal-respecting quasi-isometry $\phi: Q_{\gamma} \rightarrow$ $Q_{\gamma^{\prime}}$ coarsely respects the stable and unstable foliations of the vertical flows on $Q_{\gamma}, Q_{\gamma^{\prime}}$. Also, $\phi$ coarsely respects the patterns of singular stable and unstable leaves.

Every vertical flow line can be realized as the coarse intersection of a stable and unstable leaf, with uniform coarse intersection function independent of the choice of flow line. Proposition 5.2 and Lemma 5.1 therefore imply that $\phi$ coarsely respects the collection of vertical geodesics. A similar argument works for the collection of singular geodesics, using singular stable and unstable leaves. We record this as:

Corollary 5.3 (Vertical flow lines respected). The quasi-isometry $\phi$ of Proposition 5.2 coarsely respects the foliations of vertical flow lines in $Q_{\gamma}$ and $Q_{\gamma^{\prime}}$, as well as the patterns of singular vertical lines.

Proof of Proposition 5.2. The horizontal foliation of $Q_{\gamma}$ is an example of a uniform foliation, which means that any two leaves have finite Hausdorff distance. Any map between two horizontal leaves which moves points a bounded distance in $Q_{\gamma}$ is a quasi-isometry between those leaves. It follows that there is a canonical coarse equivalence class of quasi-isometries between any two leaves, and moreover the composition of two such quasi-isometries is another one. Each leaf is quasiisometric to the hyperbolic plane $\mathbf{H}^{2}$ and its Gromov boundary is a circle, so there is a canonical identification of all the circles at infinity to a single circle which we denote $S Q_{\gamma}$. These facts were noted by Thurston in [Thu97].

It is well-known that a quasi-isometry $\mathbf{H}^{2} \rightarrow \mathbf{H}^{2}$ induces a homeomorphism $\partial \mathbf{H}^{2} \rightarrow \partial \mathbf{H}^{2}$ between the circles at infinity, and that coarsely equivalent quasiisometries induce the same boundary map. Any horizontal respecting quasiisometry $Q_{\gamma} \rightarrow Q_{\gamma^{\prime}}$ therefore induces a homeomorphism $S Q_{\gamma} \rightarrow S Q_{\gamma^{\prime}}$ between their respective circles at infinity. The underlying idea of our proof is to find additional quasi-isometrically invariant structures on $S Q_{\gamma}$ and $S Q_{\gamma^{\prime}}$ which encode the stable and unstable foliations, and use this information to prove quasi-isometric invariance of these foliations.

To proceed with the proof we need some notation. Let $D_{t}$ denote the plane $D \times t \subset D \times \mathbf{R}=Q_{\gamma}$. The plane $D_{t}$ comes equipped with an $x y$-structure, as explained in $\S 4.4$. For each $s, t$ let $\phi_{s t}: D_{s} \rightarrow D_{t}$ be the map $(p, s) \rightarrow(p, t)$, $p \in D$. In other words, $\phi_{s t}$ flows along vertical flow segments of $Q_{\gamma}$ from $D_{s}$ to 
$D_{t}$; each such flow segment has length $|s-t|$, and so $\phi_{s t}$ is a quasi-isometry in the canonical coarse equivalence class from $D_{s}$ to $D_{t}$ as discussed above. In fact, the map $\phi_{s t}$ is an $x y$-affine homeomorphism with stretch factor $e^{|s-t|}$, implying that $\phi_{s t}$ is $e^{|s-t|}$-bilipschitz. Note that $\phi_{t u} \circ \phi_{s t}=\phi_{s u}$, for all $s, t, u \in \mathbf{R}$.

The $x y$-metric on $D_{t}$ is a $\operatorname{CAT}(0)$ metric, and it is also Gromov hyperbolic because $D_{t}$ is quasi-isometric to $\mathbf{H}^{2}$. The boundary $\partial D_{t}$ is a circle, and each quasi-isometry $\phi_{s t}: D_{s} \rightarrow D_{t}$ induces a homeomorphism $\partial \phi_{s t}: \partial D_{s} \rightarrow \partial D_{t}$.

For any two points $\xi, \eta \in \partial D_{t}$ there exists an $x y$-geodesic with endpoints $\xi, \eta$, and any two such geodesics are the boundary of an isometric embedding of $\mathbf{R} \times[a, b]$ for some $[a, b] \in \mathbf{R}$ (see [BH99]). Since $D_{t}$ is not the Euclidean plane but has a cocompact isometry group, there is an upper bound on $b-a$ independent of $\xi, \eta$. This bound is moreover independent of $t$, because coboundedness of the Teichmüller geodesic $\gamma$ and compactness of the fibers of $T^{1} \mathcal{T}$ together imply that the collection of locally CAT $(0)$ metric spaces $D_{t} / \pi_{1}(\Sigma)$ lies in a compact space of locally $\operatorname{CAT}(0)$ metrics (this is the one place in the proof where we use coboundedness of the Teichmüller geodesics in the weak convex hull of $H$ ). Let $R_{0}$ be a $t$-independent bound for $|b-a|$.

For each bi-infinite geodesic $\theta$ on $D_{0}$, the set

$$
V=V_{\theta}=\bigcup_{t \in \mathbf{R}}\left\{\phi_{0 t}(\theta)\right\}
$$

is called a vertical plane in $Q_{\gamma}$. Note that if $\partial \theta=\{\xi, \eta\} \subset \partial D_{0}$ then $\partial \phi_{0 t}(\theta)=$ $\left\{\partial \phi_{0 t}(\xi), \partial \phi_{0 t}(\eta)\right\} \subset \partial D_{t}$, and so to $V$ there is associated a unique pair of points in the circle $S Q_{\gamma}$ which we call the endpoints of $V$. Moreover, the discussion in the previous paragraph shows that the Hausdorff distance between any two vertical planes of $Q_{\gamma}$ with the same endpoints $\xi, \eta$ is at most $R_{0}$.

The first structure which $\phi: Q_{\gamma} \rightarrow Q_{\gamma^{\prime}}$ must coarsely respect is the collection of vertical planes.

Claim 5.4 (Vertical planes preserved). Let $\phi: Q_{\gamma} \rightarrow Q_{\gamma^{\prime}}$ be a horizontalrespecting quasi-isometry. If $V$ is any vertical plane in $Q_{\gamma}$, then $\phi(V)$ is a bounded Hausdorff distance from some vertical plane $V^{\prime}$ in $Q_{\gamma^{\prime}}$.

To prove Claim 5.4, let $t \rightarrow t^{\prime}$ be a bijective quasi-isometry of $\mathbf{R}$ such that $\phi\left(D_{t}\right)$ is Hausdorff close to $D_{t^{\prime}}^{\prime}$, with a uniform Hausdorff constant; we assume that the parameterizations are chosen so that $0 \rightarrow 0$ under this quasi-isometry. Composing the map $D_{t} \rightarrow \phi\left(D_{t}\right)$ with a uniformly finite distance map $\phi\left(D_{t}\right) \rightarrow D_{t^{\prime}}^{\prime}$, we obtain a quasi-isometry $\psi_{t}: D_{t} \rightarrow D_{t^{\prime}}^{\prime}$ whose quasi-isometry constants are independent of $t$.

Fix a vertical plane $V=V_{\theta}$ and for each $s$ consider the geodesic $\theta_{s}=V \cap D_{s}$. Its image quasigeodesic $\psi_{s}\left(\theta_{s}\right)$ is uniformly Hausdorff close in $D_{s^{\prime}}^{\prime}$ to some geodesic 
$\theta_{s^{\prime}}^{\prime}$, in particular $\phi_{0}\left(\theta_{0}\right)$ is close to $\theta_{0}^{\prime}$. We need only show that for each $s, \theta_{s^{\prime}}^{\prime}$ is uniformly Hausdorff close to $\phi_{0 s^{\prime}}^{\prime}\left(\theta_{0}^{\prime}\right)$, for then we can set

$$
V^{\prime}=\cup_{s^{\prime}}\left\{\phi_{0 s^{\prime}}^{\prime}\left(\theta_{0}^{\prime}\right)\right\}
$$

and it follows that $\phi(V)$ is uniformly Hausdorff close to $V^{\prime}$.

Since any two geodesics in $D_{s^{\prime}}^{\prime}$ which are Hausdorff close are $R_{0}$-Hausdorff close, we need only show that the Hausdorff distance between $\theta_{s^{\prime}}^{\prime}$ and $\phi_{0 s^{\prime}}^{\prime}\left(\theta_{0}^{\prime}\right)$ is finite, in other words these two geodesics have the same endpoints in $\partial D_{s^{\prime}}^{\prime}$. But this is an immediate consequence of the coarse commutativity of the following diagram:

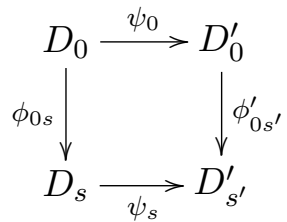

This finishes the proof of Claim 5.4. We now find finer structures which must be coarsely respected by $\phi$.

Each vertical plane $V$ comes equipped with a horizontal foliation, obtained by intersecting the plane with the horizontal foliation $\left\{D_{t}\right\}$ of $Q_{\gamma}$. Denote the leaves of this foliation by

$$
\theta_{t}=D_{t} \cap V, \quad t \in \mathbf{R}
$$

so that $\theta_{t}=\phi_{s t}\left(\theta_{s}\right)$ for any $s, t \in \mathbf{R}$. The horizontal foliation $\left\{\theta_{t}\right\}$ has a transverse orientation, pointing in the direction of increasing $t$. Note that the Hausdorff distance in $V$ between $\theta_{t}$ and $\theta_{s}$ is exactly $|t-s|$. There is a projection $\pi: V \rightarrow \mathbf{R}$ with $\theta_{t}=\pi^{-1}(t)$. Define a quasivertical line in $V$ to be a subset $L \subset V$ such that the projection $\pi: L \rightarrow \mathbf{R}$ is a quasi-isometry.

We divide the collection of vertical planes into three types: stable, unstable, and doubly unstable. A stable vertical plane is one which is contained in a leaf of the stable foliation on $Q_{\gamma}$; thus it is either a regular leaf of the stable foliation, or it is a union of two half-planes of a singular leaf. Similarly an unstable vertical plane is one contained in an unstable leaf. All other vertical planes are called doubly unstable vertical planes.

The three types of vertical planes - stable, unstable, and doubly unstablecan be distinguished from each other by horizontal respecting quasi-isometries which respect the transverse orientation, by observing the asymptotic behavior of quasivertical lines. To make this more precise, say that two quasivertical lines $L, L^{\prime} \subset P$ are upward Hausdorff close if the Hausdorff distance between $L \cap$ $\pi^{-1}[0, \infty)$ and $L^{\prime} \cap \pi^{-1}[0, \infty)$ is finite; downward Hausdorff close is similarly defined using $(-\infty, 0]$. 
Claim 5.5. Let $V$ be a vertical plane. Then:

Stable: If $V$ is a stable plane, then any two quasivertical lines in $V$ are upward Hausdorff close but not downward Hausdorff close.

Unstable: If $V$ is an unstable plane, then any two quasivertical lines in $V$ are downward Hausdorff close but not upward Hausdorff close.

Doubly unstable: If $V$ is a doubly unstable plane then there exist two quasivertical lines in $V$ which are neither upward Hausdorff close nor downward Hausdorff close.

To prove Claim 5.5 when $V$ is stable or unstable, observe first that $V$ with its horizontal foliation $\left\{\theta_{t}\right\}$ is isometric to the hyperbolic plane $\mathbf{H}^{2}$ in the upper half plane model, with the "horizontal" horocyclic foliation centered on the point $\infty$. If $V$ is stable (resp. unstable) then the transverse orientation points towards $\infty$ (resp. away). Next observe that any quasivertical line in $\mathbf{H}^{2}$ is a quasigeodesic with one endpoint at $\infty$, and so any two quasivertical lines are Hausdorff close in the direction of $\infty$.

To prove Claim 5.5 when $V$ is doubly unstable, observe that $\theta_{0}=V \cap D_{0}$ is an $x y$-geodesic in $D_{0}$ which is not contained in a leaf of either the $x$-foliation or the $y$-foliation on $D_{0}$. There exists, therefore, two points $p, q \in \theta_{0}$ which do not lie in the same leaf of either the $x$-foliation or the $y$-foliation on $D_{0}$. The vertical flow lines $p \cdot \mathbf{R}, q \cdot \mathbf{R}$ in $Q_{\gamma}$ are evidently neither upward nor downward Hausdorff close in the singular SOLV-metric on $Q_{\gamma}$, and so the same is true in $V$. This proves Claim 5.5.

The same discussion holds, of course, in $Q_{\gamma^{\prime}}$. Now consider a horizontalrespecting quasi-isometry $\phi: Q_{\gamma} \rightarrow Q_{\gamma^{\prime}}$. By reversing the upward orientation in $Q_{\gamma^{\prime}}$ if necessary, we may assume that $\phi$ respects the upward orientation. Let $V$ be any vertical plane in $Q_{\gamma}$. We have shown in Claim 5.4 that $\phi(V)$ is Hausdorff close to some vertical plane $V^{\prime}$ in $Q_{\gamma^{\prime}}$. Composing the map $V \rightarrow \phi(V)$ with any finite distance map $\phi(V) \rightarrow V^{\prime}$, we obtain therefore a quasi-isometry $\psi: V \rightarrow V^{\prime}$. Each horizontal leaf of $V$ (resp. $V^{\prime}$ ) is a coarse intersection of $V$ with a horizontal leaf of $Q_{\gamma}$ (resp. $Q_{\gamma^{\prime}}$ ), and it follows that $\psi$ coarsely respects the horizontal foliations and their transverse orientations.

Claim 5.5 shows manifestly that stable vertical planes are coarsely respected by $\phi: Q_{\gamma} \rightarrow Q_{\gamma^{\prime}}$, and similarly for unstable vertical planes. To finish the proof of Proposition 5.2, given two stable (resp. unstable) vertical planes $V_{1}, V_{2}$ in $Q_{\gamma}$ or in $Q_{\gamma^{\prime}}$, we must give a quasi-isometrically invariant property which characterizes $V_{1}, V_{2}$ lying in the same weak stable (resp. unstable) leaf. Namely, $V_{1}, V_{2}$ lie in the same leaf if and only if, for any $t \in \mathbf{R}$, the triple coarse intersection $V_{1} \cap_{c} V_{2} \cap_{c} D_{t}$ is unbounded. 
Remark. There is an alternative "dynamical" proof of Proposition 5.2 which we worked out first, and which parallels the proof of the analogous proposition in [FM00a]. The latter is concerned with the solvable Lie groups associated to geodesics in the symmetric space of $\operatorname{GL}(n, \mathbf{R})$, and the natural Anosov flows on these solvable Lie groups; the proof in [FM00a] is an easy consequence of the shadowing lemma for Anosov flows. Unfortunately the shadowing lemma is false in pseudo-Anosov dynamics, complicating the situation drastically. There is an alternative shadowing theory for pseudo-Anosov dynamical systems, developed in [Han85], [Han88], and [Mos89], and this was used in our original proof of Proposition 5.2. The proof given above for Proposition 5.2 entirely avoids these issues by using Gromov hyperbolicity of the horizontal leaves - a fact which was not available in [FM00a], where the horizontal leaves are Euclidean.

\section{Periodic hyperplanes}

A hyperplane $P_{w}$ in $X_{H}$ is a periodic hyperplane if $w$ is a periodic geodesic in $T_{H}$, that is, the subgroup $C_{w}$ of $H$ stabilizing $w$ is infinite cyclic. Periodic hyperplanes $P_{w}$ are special in that they admit a priori extra isometries, above and beyond the isometric action of $\pi_{1}(\Sigma)$ : the subgroup $\pi_{1}(\Sigma) \rtimes C_{w}$ of $\Gamma_{H}$ acts isometrically on $P_{w}$.

Our goal now is to show that horizontal-respecting quasi-isometries of periodic hyperplanes must remember the extra symmetries.

Recall that $w \leftrightarrow \gamma_{w}$ is a bijection between geodesics in the tree $T_{H}$ and Teichmüller geodesics in $\mathcal{H} \Lambda$.

Proposition 6.1 (Periodic hyperplanes preserved). Given $w, w^{\prime}$ geodesics in $T_{H}$, suppose that there exists a horizontal respecting quasi-isometry between hyperplanes $\phi: P_{w} \rightarrow P_{w^{\prime}}$. Then:

(1) $w$ is periodic if and only if $w^{\prime}$ is periodic.

(2) If $w, w^{\prime}$ are periodic, and if $\gamma, \gamma^{\prime} \subset \mathcal{H} \Lambda$ are the geodesics in $\mathcal{T}$ associated to $w, w^{\prime}$ respectively, then $\gamma, \gamma^{\prime}$ are periodic and there is a singular SOLVisometry $\Phi: Q_{\gamma} \rightarrow Q_{\gamma^{\prime}}$ such that the following diagram coarsely commutes:

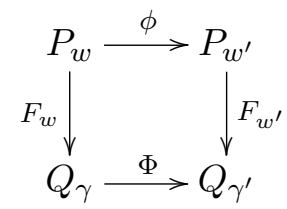

That is, $d\left(F_{w^{\prime}}(\phi(x)), \Phi\left(F_{w}(x)\right)\right) \leq A$ where $A$ depends only on the quasiisometry constants of $\phi$, not on $w$ or $w^{\prime}$. 
We start by reducing the proposition to a statement about singular SOLVmanifolds by using the following result, which shows that various competing notions of "periodicity" are in fact equivalent:

Lemma 6.2 (Characterizing periodicity). Given a $T_{H}$ geodesic $w$ and the corresponding Teichmüller geodesic $\gamma=\gamma_{w}$, the following are equivalent:

(1) $P_{w}$ is periodic, meaning that the group $C_{w}$ is infinite.

(2) $\gamma$ is periodic in $\mathcal{T}$, meaning that the group $\operatorname{Stab}_{\mathcal{T}}(\gamma)$ is infinite.

(3) $Q_{\gamma}$ is periodic, meaning that the group $C_{\gamma}=\operatorname{Isom}\left(Q_{\gamma}\right) / \operatorname{Isom}_{h}\left(Q_{\gamma}\right)$ is infinite.

Proof. We clearly have inclusions $C_{w} \subset \operatorname{Stab}_{\mathcal{T}}\left(\gamma_{w}\right) \subset C_{\gamma}$ and so (1) implies (2) implies (3).

To prove (2) implies (1), suppose that $\gamma$ is periodic in $\mathcal{T}$, that is, $\gamma$ is the axis of some pseudo-Anosov element $\phi \in \mathcal{M}$. Choose a point $p \in \gamma$, and consider the sequence $\phi^{n}(p), n>0$. Since $H$ acts cocompactly on $\mathcal{H} \Lambda$, there is a sequence $\psi_{n} \in H$ such that $\left\{\psi_{n} \circ \phi^{n}(p) \mid n>0\right\}$ is a bounded subset of $\mathcal{T}$. Since $\mathcal{M}$ acts properly on $\mathcal{T}$, there exist $m>n>0$ such that $\psi_{n} \circ \phi^{n}=\psi_{m} \circ \phi^{m}$. It follows that $\phi^{m-n}=\psi_{m}^{-1} \psi_{n} \in H$, and so $w$ is periodic in $T_{H}$.

To prove (3) implies (2), choose a horizontal leaf $D_{\gamma}$ of $Q_{\gamma}$ and so we have the split exact sequence

$$
1 \rightarrow \operatorname{Isom}_{+}\left(D_{\gamma}\right) \approx \operatorname{Isom}_{h}\left(Q_{\gamma}\right) \rightarrow \operatorname{Isom}_{+}\left(Q_{\gamma}\right) \rightarrow C_{\gamma+} \rightarrow 1
$$

where $C_{\gamma+}$ is an infinite cyclic subgroup of index $\leq 2$ in $C_{\gamma}$. We also have a finite index inclusion $\pi_{1}(\Sigma)<\operatorname{Isom}_{+}\left(D_{\gamma}\right)$. Since $C_{\gamma+}$ acts by automorphisms of $\operatorname{Isom}_{+}\left(D_{\gamma}\right)$ it follows that $C_{\gamma+}$ has a finite index subgroup stabilizing $\pi_{1}(\Sigma)$; this subgroup is clearly identified with a subgroup of $\operatorname{Stab}_{\mathcal{T}}\left(\gamma_{w}\right)$ and so the latter is infinite.

Note from the proof that each of the inclusions $C_{w} \subset \operatorname{Stab}_{\mathcal{T}}\left(\gamma_{w}\right) \subset C_{\gamma}$ has finite index; examples may be constructed in which any number of these inclusions is proper.

By combining Lemma 6.2 with Proposition 4.4, it follows immediately that Proposition 6.1 is reduced to the following:

Lemma 6.3. Given geodesics $\gamma, \gamma^{\prime}$ in $\mathcal{T}$, if $\gamma$ is periodic, and if there exists a horizontal respecting quasi-isometry $Q_{\gamma} \rightarrow Q_{\gamma^{\prime}}$, then $\gamma^{\prime}$ is periodic and there exists a singular SOLV isometry $Q_{\gamma} \rightarrow Q_{\gamma^{\prime}}$. 
Remark. Our proof of Lemma 6.3 uses both Thurston's hyperbolization theorem for mapping tori of pseudo-Anosov homeomorphisms [Ota96] as well as the geodesic pattern rigidity theorem of R. Schwartz [Sch97], and to apply these results we need to invoke periodicity of $\gamma$. Existence of a horizontal respecting quasi-isometry between $Q_{\gamma}$ and $Q_{\gamma^{\prime}}$ ought to imply existence of a singular SOLVisometry, without assuming periodicity of $\gamma$; however, we do not know a proof.

\subsection{Rigidity of periodic hyperplanes}

The main step in the proof of Lemma 6.3 is Proposition 6.4 below, which shows that a periodic singular SOLV-manifold $Q_{\gamma}$ has the rigidity property that its horizontal respecting quasi-isometry group equals its isometry group. Here is the basic setup.

For each periodic geodesic $\gamma$ in $\mathcal{T}$, fix a base horizontal leaf $D_{\gamma} \subset Q_{\gamma}$. Recall that $\pi_{1}(\Sigma)$ acts as a deck transformation group on $D_{\gamma}$. The singular SOLV-metric on $Q_{\gamma}$ induces an $x y$-structure on $D_{\gamma}$ with respect to which we have an inclusion $\pi_{1}(\Sigma) \hookrightarrow \operatorname{Isom}_{+}\left(D_{\gamma}\right)$. There is a commutative diagram of short exact sequences

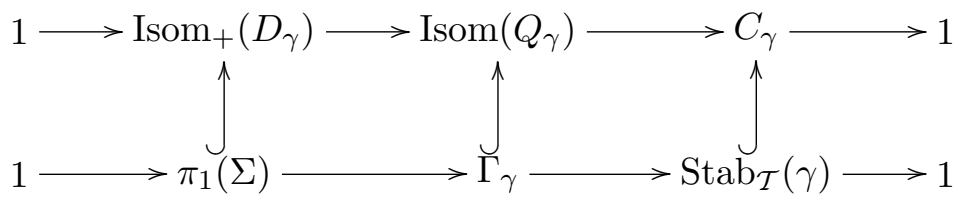

where each vertical arrow is a finite index inclusion. The group $C_{\gamma}$ is either $\mathbf{Z}$ or $D_{\infty}$. The quotient $Q_{\gamma} / \operatorname{Isom}\left(Q_{\gamma}\right)$ is a closed 3-dimensional orbifold, equipped with an orbifold fibration over the circle (when $C_{\gamma}=\mathbf{Z}$ ) or the interval orbifold (when $\left.C_{\gamma}=D_{\infty}\right)$; in the latter case we choose $D_{\gamma}$ so that it projects to a generic point of the interval orbifold, i.e. so that the stabilizer of $D_{\gamma}$ in $\operatorname{Isom}\left(Q_{\gamma}\right)$ equals $\operatorname{Isom}_{+}\left(D_{\gamma}\right)$. It follows that the generic fiber of the quotient 3-orbifold $Q_{\gamma} / \operatorname{Isom}\left(Q_{\gamma}\right)$ is the closed 2-orbifold $\mathcal{O}_{\gamma}=D_{\gamma} / \operatorname{Isom}_{+}\left(D_{\gamma}\right)$ equipped with the quotient $x y$-structure.

Note that the quotient 3-orbifold $Q_{\gamma} / \Gamma_{\gamma}$ also fibers over the circle or the interval orbifold, with fiber $\Sigma$. There is a finite covering map $\Sigma \rightarrow \mathcal{O}_{\gamma}$ and the fiber monodromy map on $\mathcal{O}_{\gamma}$ lifts to a pseudo-Anosov homeomorphism $\phi: \Sigma \rightarrow \Sigma$.

Let $\mathrm{QI}_{h}\left(Q_{\gamma}\right)$ be the subgroup of $\mathrm{QI}\left(Q_{\gamma}\right)$ represented by horizontal respecting quasi-isometries of $Q_{\gamma}$. Clearly there is a homomorphism

$$
i: \operatorname{Isom}\left(Q_{\gamma}\right) \rightarrow \mathrm{QI}_{h}\left(Q_{\gamma}\right)
$$

Proposition 6.4 (Rigidity of periodic hyperplanes). If $\gamma$ is periodic then the homomorphism $i: \operatorname{Isom}\left(Q_{\gamma}\right) \rightarrow \mathrm{QI}_{h}\left(Q_{\gamma}\right)$ is an isomorphism. 
Proof. A nontrivial isometry $\phi$ of $Q_{\gamma}$ must map some vertical geodesic to a different vertical geodesic. Since any two vertical geodesics in $Q_{\gamma}$ have infinite Hausdorff distance, $\phi$ is an infinite distance from the identity, so that $i$ is injective (injectivity of $i$ therefore is true regardless of periodicity).

We now prove that $i$ is surjective: every horizontal respecting quasi-isometry of $Q_{\gamma}$ is a bounded distance from an isometry.

Thurston's geometrization theorem for pseudo-Anosov mapping tori gives a hyperbolic structure on the 3-dimensional orbifold $Q_{\gamma} / \operatorname{Isom}\left(Q_{\gamma}\right)$. This yields a properly discontinuous, cocompact, isometric, faithful action

$$
h: \operatorname{Isom}\left(Q_{\gamma}\right) \rightarrow \operatorname{Isom}\left(\mathbf{H}^{3}\right)
$$

and a quasi-isometric, $h$-equivariant homeomorphism

$$
q: Q_{\gamma} \rightarrow \mathbf{H}^{3}
$$

For each singular vertical geodesic $\ell \subset Q_{\gamma}$, the image $q(\ell)$ is a quasigeodesic in $\mathbf{H}^{3}$, which by the Morse-Mostow Lemma is a bounded Hausdorff distance (not depending on $\ell$ ) from a unique geodesic in $\mathbf{H}^{3}$; let $L$ denote the set of all such geodesics in $\mathbf{H}^{3}$. Let $\operatorname{Isom}\left(\mathbf{H}^{3}, L\right)$ be the group of isometries of $\mathbf{H}^{3}$ which permute the collection $L$. Also, let $F$ be the foliation of $\mathbf{H}^{3}$ obtained by pushing forward via $q$ the horizontal foliation of $Q_{\gamma}$, and let $\operatorname{Isom}\left(\mathbf{H}^{3}, L, F\right)$ be the subgroup of $\operatorname{Isom}\left(\mathbf{H}^{3}, L\right)$ which coarsely respects $F$. Note that image $(h) \subset \operatorname{Isom}\left(\mathbf{H}^{3}, L, F\right)$. Now we show that $h$ factors through $i$.

For each horizontal respecting quasi-isometry $\psi$ of $Q_{\gamma}$, we obtain a quasiisometry $q \psi q^{-1}$ of $\mathbf{H}^{3}$, inducing a homomorphism $\hat{q}: \mathrm{QI}_{h}\left(Q_{\gamma}\right) \rightarrow \mathrm{QI}\left(\mathbf{H}^{3}\right)$. Since $\psi$ coarsely respects singular vertical geodesics and horizontal leaves in $Q_{\gamma}$, it follows that $q \psi q^{-1}$ coarsely respects $L$ and $F$.

Since $q \psi q^{-1}$ coarsely respects $L$, since $L$ is invariant under the cocompact isometry group image $(h)$, and since there are only finitely many orbits of the action of image $(h)$ on $L$, we may directly apply the main theorem of [Sch97] which says in this setting that $q \psi q^{-1}$ is a bounded distance (not uniformly so) from a unique isometry of $\mathbf{H}^{3}$ which strictly respects $L$.

It follows that the image of $\hat{q}$ is contained in $\operatorname{Isom}\left(\mathbf{H}^{3}, L\right)$, and in fact in $\operatorname{Isom}\left(\mathbf{H}^{3}, L, F\right)$. It's evident that the composition

$$
\operatorname{Isom}\left(Q_{\gamma}\right) \stackrel{i}{\rightarrow} \mathrm{QI}_{h}\left(Q_{\gamma}\right) \stackrel{\hat{q}}{\rightarrow} \operatorname{Isom}\left(\mathbf{H}^{3}, L, F\right) \subset \operatorname{Isom}\left(\mathbf{H}^{3}\right)
$$

is identical with the homomorphism $h$. The homomorphism $\hat{q}$ is obviously injective, and so to prove surjectivity of $i$ it suffices to show that image $(h)=$ $\operatorname{Isom}\left(\mathbf{H}^{3}, L, F\right)$. 
Consider the short exact sequence

$$
1 \rightarrow \operatorname{Isom}_{+}\left(D_{\gamma}\right) \rightarrow \operatorname{Isom}\left(Q_{\gamma}\right) \rightarrow C_{\gamma} \rightarrow 1
$$

Since $\operatorname{Isom}_{+}\left(D_{\gamma}\right)$ is normal in $\operatorname{Isom}\left(Q_{\gamma}\right)$, and since $\operatorname{Isom}\left(Q_{\gamma}\right)$ is identified via $h$ with a finite index subgroup of $\operatorname{Isom}\left(\mathbf{H}^{3}, L, F\right)$ (both being discrete and cocompact on $\left.\mathbf{H}^{3}\right)$, it follows that $\operatorname{Isom}_{+}\left(D_{\gamma}\right)$ has a normalizer of finite index in $\operatorname{Isom}\left(\mathbf{H}^{3}, L, F\right)$; choose coset representatives $g_{1}, \ldots, g_{n}$ of the normalizer. Each leaf of $F$ is coarsely equivalent to $\operatorname{Isom}_{+}\left(D_{\gamma}\right)$, and $g_{1}, \ldots, g_{n}$ coarsely respects $F$, and so each conjugate subgroup

$$
g_{1} \operatorname{Isom}_{+}\left(D_{\gamma}\right) g_{1}^{-1}, \ldots, g_{n} \operatorname{Isom}_{+}\left(D_{\gamma}\right) g_{n}^{-1}
$$

is coarsely equivalent to $\operatorname{Isom}_{+}\left(D_{\gamma}\right)$.

Now we apply an elementary lemma of [MSW00] which says that for a finite collection of subgroups in a finitely generated group, the coarse intersection of those subgroups is coarsely equivalent to their intersection. The intersection of the above conjugates of $\operatorname{Isom}_{+}\left(D_{\gamma}\right)$ is therefore coarsely equivalent to $D_{\gamma}$.

Another elementary lemma of [MSW00] says that in a finitely generated group, given subgroups $A \subset B$, if $A, B$ are coarsely equivalent, then $A$ has finite index in $B$. It follows that the intersection of the conjugates of $\operatorname{Isom}_{+}\left(D_{\gamma}\right)$ in $\operatorname{Isom}\left(\mathbf{H}^{3}, L, F\right)$ has finite index in $\operatorname{Isom}_{+}\left(D_{\gamma}\right)$. Thus we obtain a normal subgroup $N \subset \operatorname{Isom}\left(\mathbf{H}^{3}, L, F\right)$ of finite index in $\operatorname{Isom}_{+}\left(D_{\gamma}\right)$. The quotient group $\operatorname{Isom}\left(\mathbf{H}^{3}, L, F\right) / N$ is a finite index supergroup of $C_{\gamma}=\operatorname{Isom}\left(Q_{\gamma}\right) / \operatorname{Isom}_{+}\left(D_{\gamma}\right)$, and so the quotient is virtually cyclic. Since $\operatorname{Isom}\left(\mathbf{H}^{3}, L, F\right)$ is a 3-orbifold group it follows that the quotient $\operatorname{Isom}\left(\mathbf{H}^{3}, L, F\right) / N$ is either $\mathbf{Z}$ or $D_{\infty}$.

The orbifold $\mathbf{H}^{3} / \operatorname{Isom}\left(\mathbf{H}^{3}, L, F\right)$ therefore fibers over the circle or the interval orbifold, with generic fiber $\mathcal{O}$, and with $\pi_{1}(\mathcal{O})$ identified with $N$. Lifting this fibration to $\mathbf{H}^{3}$ we obtain an $\operatorname{Isom}\left(\mathbf{H}^{3}, L, F\right)$ equivariant fibration coarsely equivalent to $F$. We may therefore replace $F$ with this fibration, and so $F$ is strictly invariant under $\operatorname{Isom}\left(\mathbf{H}^{3}, L, F\right)$. The monodromy map on $\mathcal{O}$ is pseudo-Anosov. There is a finite index covering map $Q_{\gamma} / \operatorname{Isom}\left(Q_{\gamma}\right) \rightarrow \mathbf{H}^{3} / \operatorname{Isom}\left(\mathbf{H}^{3}, L, F\right)$, taking fibration to fibration. By uniqueness of pseudo-Anosov homeomorphisms in their isotopy classes $\left[\mathrm{FLP}^{+} 79\right]$, it follows that the stable and unstable measured foliations for the monodromy map of $\mathcal{O}$ lift to the stable and unstable measured foliations for the monodromy map on the generic fiber $\mathcal{O}_{\gamma}$ of $Q_{\gamma} / \operatorname{Isom}\left(Q_{\gamma}\right)$. But this shows that $\operatorname{Isom}\left(\mathbf{H}^{3}, L, F\right)$ acts isometrically on $Q_{\gamma}$, proving that image $(h)=\operatorname{Isom}\left(\mathbf{H}^{3}, L, F\right)$.

This proves Proposition 6.4.

\subsection{Proof of Lemma 6.3}

Assume $Q_{\gamma}$ is periodic and $\phi: Q_{\gamma} \rightarrow Q_{\gamma^{\prime}}$ is a horizontal respecting quasi-isometry. We'll construct an isometry $\Phi: Q_{\gamma} \rightarrow Q_{\gamma^{\prime}}$, the existence of which implies that $Q_{\gamma^{\prime}}$ 
is periodic.

Applying Proposition 5.2, we may move $\phi$ a bounded distance so that $\phi$ is a homeomorphism, respecting the horizontal foliations and the weak stable and unstable foliations. We may assume that the base horizontal leaves $D_{\gamma} \subset Q_{\gamma}$, $D_{\gamma^{\prime}} \subset Q_{\gamma^{\prime}}$ are chosen so that $\phi\left(D_{\gamma}\right)=D_{\gamma^{\prime}}$.

Let $A_{\gamma}: \pi_{1}(\Sigma) \rightarrow \operatorname{Isom}\left(Q_{\gamma}\right)$ and $A_{\gamma^{\prime}}: \pi_{1}(\Sigma) \rightarrow \operatorname{Isom}\left(Q_{\gamma^{\prime}}\right)$ be the standard isometric actions, preserving each horizontal leaf. Let $\widehat{\mathrm{QI}}_{h}\left(Q_{\gamma}\right)$ be the subsemigroup of $\widehat{\mathrm{QI}}\left(Q_{\gamma}\right)$ that coarsely respects the horizontal foliation of $Q_{\gamma}$, and let

$$
B_{\gamma}=\phi^{-1} \circ A_{\gamma^{\prime}} \circ \phi: \pi_{1}(\Sigma) \rightarrow \widehat{\mathrm{QI}}_{h}\left(Q_{\gamma}\right)
$$

be the conjugated action (its really an action, not just a quasi-action, because $\phi$ is a homeomorphism). Note that $B_{\gamma}$ preserves each horizontal leaf of $Q_{\gamma}$ as well as the strong stable and unstable foliations in that leaf; however, $B_{\gamma}$ does not a priori preserve the invariant measures on those foliations.

Applying Proposition 6.4, we conclude that $B_{\gamma}$ is a bounded distance from an isometric action, that is, there exists a homeomorphism $\xi: Q_{\gamma} \rightarrow Q_{\gamma}$ which moves each point a uniformly bounded distance, such that $\xi^{-1} \circ B_{\gamma} \circ \xi$ is an isometric action of $\pi_{1}(\Sigma)$ on $Q_{\gamma}$. The action $\xi^{-1} \circ B_{\gamma} \circ \xi$ also preserves each horizontal leaf of $Q_{\gamma}$ and the strong stable and unstable foliations in that leaf.

We claim that $B_{\gamma}=\xi^{-1} \circ B_{\gamma} \circ \xi$. Consider a horizontal leaf $L$ of $Q_{\gamma}$ and a point $x \in L$. Let $\ell^{s}, \ell^{u}$ be the strong stable and unstable leaves in $L$ passing through $x$, and so $x=\ell^{s} \cap \ell^{u}$. Consider an element $\beta \in B_{\gamma}\left(\pi_{1}(\Sigma)\right)$. We know that $\beta(L)=\xi^{-1} \beta \xi(L)=L$. We also know that $\beta\left(\ell^{s}\right)$ and $\xi^{-1} \beta \xi\left(\ell^{s}\right)$ are both strong stable leaves in $L$, and they are a bounded distance from each other, implying that $\beta\left(\ell^{s}\right)=\xi^{-1} \beta \xi\left(\ell^{s}\right)$. Similarly, $\beta\left(\ell^{u}\right)=\xi^{-1} \beta \xi\left(\ell^{u}\right)$. Therefore, $\beta(x)=\xi^{-1} \beta \xi(x)$, proving the claim.

It follows from the claim that $B_{\gamma}$ does, in fact, preserve the invariant measures on the strong stable and unstable foliations in each horizontal leaf of $Q_{\gamma}$.

Fix a horizontal leaf $L \subset Q_{\gamma}$ and let $L^{\prime}=\phi(L) \subset Q_{\gamma^{\prime}}$. So $\phi$ takes the strong stable and unstable foliations in $L$ to those in $L^{\prime}$. Let $f^{s}, f^{u}$ be the strong stable and unstable measured foliations in $L$, and let $f^{\prime s}, f^{\prime u}$ the strong stable and unstable measured foliations in $L^{\prime}$. The map $\phi$ pushes the transverse measures on $f^{s}, f^{u}$ forward to new transverse measures on $f^{\prime s}, f^{\prime u}$.

Since $B_{\gamma}=\phi^{-1} A_{\gamma^{\prime}} \phi$ acts isometrically on the transverse measures of $f^{s}, f^{u}$, it follows that $A_{\gamma^{\prime}}$ acts isometrically on both the old and the new transverse measures on $f^{\prime s}, f^{\prime u}$. However, $f^{\prime s}, f^{\prime u}$ are uniquely ergodic with respect to the $A_{\gamma^{\prime}}$ action, i.e. they have projectively unique transverse measures invariant under the $A_{\gamma^{\prime}}$ action. This follows from the fact that the Teichmüller geodesic $\gamma^{\prime}$ is cobounded, together with a theorem of $\mathrm{H}$. Masur that if $\xi \in \mathbf{P} \mathcal{M} F$ is not uniquely ergodic 
then any Teichmüller ray with ending foliation $\xi$ is not cobounded [Mas80]. Hence the old and new transverse measures on $f^{\prime s}, f^{\prime u}$ differ by multiplicative constants. These multiplicative constants are inverses to each other, because the action $A_{\gamma^{\prime}}$ has cofinite area. It follows that $\phi$ restricts to an $x y$-affine isomorphism from $D_{\gamma}$ to $D_{\gamma^{\prime}}$. This implies in turn that the quasi-isometry $\phi: Q_{\gamma} \rightarrow Q_{\gamma^{\prime}}$ may be altered a bounded amount, moving each point a fixed amount up or down in its vertical geodesic, to obtain an isometry $\Phi: Q_{\gamma} \rightarrow Q_{\gamma^{\prime}}$.

It follows that $D_{\gamma^{\prime}}$ has an extra, nonisometric, affine symmetry, and so $\gamma^{\prime}$ is periodic.

This completes the proof of Lemma 6.3 and therefore also of Proposition 6.1.

\section{The endgame: computing $\mathrm{QI}\left(\Gamma_{H}\right)$ and $\operatorname{Comm}\left(\Gamma_{H}\right)$}

\subsection{An injection $\mathrm{QI}\left(X_{H}\right) \rightarrow \operatorname{QSym}\left(S^{1}\right)$}

Let $\operatorname{QSym}\left(S^{1}\right)$ denote the group of quasisymmetric homeomorphisms of the circle $S^{1}$. It is well-known that the extension of a quasi-isometry of $\mathbf{H}^{2}$ to $S^{1}$ induces an isomorphism $\mathrm{QI}\left(\mathbf{H}^{2}\right) \approx \mathrm{QSym}\left(S^{1}\right)$ : boundary extension of quasi-isometries defines an injection from the quasi-isometry group of any word hyperbolic group to the homeomorphism group of its boundary; and quasi-symmetric homeomorphisms of $S^{1}$ are exactly the extensions of quasi-isometries of $\mathbf{H}^{2}$. The key to all of this is the theorem of Ahlfors and Beurling [AB56] that quasisymmetric homeomorphisms of $S^{1}$ are exactly the extensions of quasiconformal homeomorphisms of the unit disc.

Fix once and for all a base fiber $D_{0}=\pi^{-1}\left(\tau_{0}\right)$ of $X_{H}, \tau_{0} \in T_{H}$. Identify $D_{0}$ with $\mathbf{H}^{2}$, so $\partial D_{0}$ is identified with $S^{1}$. Then there is a map $\Psi: \operatorname{QI}\left(X_{H}\right) \rightarrow \operatorname{QSym}\left(S^{1}\right)$, defined as follows. Given a quasi-isometry $f: X_{H} \rightarrow X_{H}$, the image $f\left(D_{0}\right)$ is Hausdorff close to some fiber $D^{\prime}$. Consider the composition

$$
D_{0} \rightarrow f\left(D_{0}\right) \rightarrow D^{\prime} \rightarrow D_{0}
$$

where the first map is $f$ and the other maps are closest point projections. Then the composition $D_{0} \rightarrow D_{0}$ is clearly a quasi-isometry, and so induces an element $\operatorname{QSym}\left(S^{1}\right)$. Hence we have a map $\Psi: \operatorname{QI}\left(X_{H}\right) \rightarrow \operatorname{QSym}\left(S^{1}\right)$, which is easily seen to be well-defined, and in fact a homomorphism.

Proposition 7.1. The homomorphism $\Psi: \operatorname{QI}\left(X_{H}\right) \rightarrow \operatorname{QSym}\left(S^{1}\right)$ is injective.

Proof. By Propositions 4.1 and 4.2, and the fact that the projection $X_{H} \rightarrow T_{H}$ induces an isometry between the space of horizontal leaves of $X_{H}$ with the Hausdorff metric and the quotient tree $T_{H}$, it follows that any quasi-isometry $f: X_{H} \rightarrow X_{H}$ induces a quasi-isometry of $T_{H}$ and so $f$ induces a homeomorphism $\Xi(f): \Lambda \rightarrow \Lambda$ of the Cantor set $\Lambda=\partial T_{H} \subset \mathbf{P} \mathcal{M} F$. 
Suppose that $\Psi(f) \in \operatorname{QSym}\left(S^{1}\right)$ is the identity map on $S^{1}$.

We claim that the induced map $\Xi(f): \Lambda \rightarrow \Lambda$ is the identity. It follows that the induced quasi-isometry $T_{H} \rightarrow T_{H}$ is a bounded distance from the identity, and so $f$ takes each horizontal leaf of $X_{H}$ a bounded distance from itself; and since the induced boundary map of that leaf is the identity, it follows that $f$ takes each point a bounded distance from itself, proving the proposition.

For proving the claim (and for later purposes) we review the well-known embedding of $\mathcal{M F}$ into the space of $\pi_{1}(\Sigma)$-invariant measures on the "Möbius band beyond infinity" of the hyperbolic plane. That is, consider the double set of the circle, $D S^{1}=\left\{\{x, y\} \subset S^{1} \mid x \neq y\right\}$; with respect to the Klein model $D_{0}=\mathbf{H}^{2} \subset \mathbf{R P}^{2}$, the usual duality gives a bijection between $D S^{1}$ and the Möbius band beyond infinity $\mathbf{R} \mathbf{P}^{2}-\overline{\mathbf{H}}^{2}$.

Let $M\left(D S^{1}\right)$ be the space of Borel measures on $D S^{1}$ with the weak ${ }^{*}$ topology, and let $\mathbf{P} M\left(D S^{1}\right)$ be the space of projective classes of elements of $M\left(D S^{1}\right)$. The space $\mathcal{M F}$ embeds into the space of $\pi_{1}(\Sigma)$-invariant elements of $M\left(D S^{1}\right)$, by lifting a measured foliation on $\Sigma$ to a $\pi_{1}(\Sigma)$-invariant measured foliation on $D_{0}$, and then identifying each leaf of the lifted foliation with the correspond pair of endpoints in $D S^{1}$. The space $\mathbf{P} \mathcal{M} F$ therefore embeds as $\pi_{1}(\Sigma)$-invariant elements of $\mathbf{P} M\left(D S^{1}\right)$. Given $b \in \mathbf{P} \mathcal{M} F$ let $\mu_{b} \in \mathbf{P} M\left(D S^{1}\right)$ be the corresponding projective class of measures, let $\operatorname{supp}\left(\mu_{b}\right) \subset D S^{1}$ be the support, and let $E_{b}=\left|\operatorname{supp}\left(\mu_{b}\right)\right|$ be the union of all the pairs in $\operatorname{supp}\left(\mu_{b}\right)$. Note that $E_{b}$ may also be described as the endpoint set of $b$, the set of endpoints in $S^{1}$ of the leaves of the measured foliation $\widetilde{\mathcal{F}}_{b}$ on $D_{0}$ obtained by lifting any measured foliation $\mathcal{F}_{b}$ on $\Sigma$ that represents $b$.

By Proposition 5.2, for all $b \in \Lambda$ and all quasi-isometries $\phi$ of $X_{H}$ we have

$$
\Psi(\phi)\left(E_{b}\right)=E_{\Xi(\phi)(b)}
$$

From our assumption that $\Psi(f)$ is the identity on $S^{1}$ it follows that $\Psi(f)\left(E_{b}\right)=$ $E_{b}$, for all $b \in \Lambda$. Note however that if $b \neq b^{\prime} \in \Lambda$ then $E_{b} \cap E_{b^{\prime}}=\emptyset$, because the projective classes of $\mathcal{F}_{b}, \mathcal{F}_{b^{\prime}}$ in $\mathbf{P} \mathcal{M} F$ are connected by a Teichmüller geodesic in $\mathcal{H} \Lambda$, and so the measured foliations $\mathcal{F}_{b}, \mathcal{F}_{b^{\prime}}$ can be chosen to be transverse in $\Sigma$; the lifted foliations $\widetilde{\mathcal{F}}_{b}, \widetilde{\mathcal{F}}_{b^{\prime}}$ are therefore transverse in $D_{0}$ and so their endpoint sets $E_{b}, E_{b^{\prime}}$ in $S^{1}$ are disjoint. It follows that $\Xi(f)(b)=b$ for all $b \in \Lambda$.

\subsection{The orbifold $\mathcal{O}_{H}$ associated to a Schottky group $H$}

Our goal in this subsection and the next is to compute the quasi-isometry group $\mathrm{QI}\left(\Gamma_{H}\right)$. Using the injection $\Psi: \mathrm{QI}\left(\Gamma_{H}\right) \rightarrow \operatorname{QSym}\left(S^{1}\right)$ provided by Proposition 7.1, our computation will consist of an explicit description of the subgroup $\Psi\left(\mathrm{QI}\left(\Gamma_{H}\right)\right)<\operatorname{QSym}\left(S^{1}\right)$; without further mention we shall identify $\mathrm{QI}\left(\Gamma_{H}\right)$ with this subgroup. 
The first step in the computation of $\mathrm{QI}\left(\Gamma_{H}\right)$ is to find a natural orbifold subcover $\Sigma \rightarrow \mathcal{O}_{H}$ associated to a Schottky subgroup $H<\mathcal{M}(\Sigma)$. The orbifold $\mathcal{O}_{H}$ is an important invariant of $H$; it is the smallest subcover of $\Sigma$ to which $H$ descends as a subgroup of $\mathcal{M}\left(\mathcal{O}_{H}\right)$.

Recall we have fixed a base fiber $D_{0}$ of $X_{H}$ over a base point $\tau_{0} \in T_{H}$, and we identify $D_{0}$ with the universal cover $\widetilde{\Sigma}$.

To each periodic hyperplane $P_{w}$ we associate a finite-index supergroup of $\pi_{1}(\Sigma)$ in $\operatorname{QSym}\left(S^{1}\right)$, as follows. In the associated singular SOLV-manifold $Q_{\gamma_{w}}$ pick any horizontal leaf $D_{w}$, which has an induced $x y$ structure with $x y$-affine automorphism group denoted $\operatorname{Aff}\left(D_{w}\right)$. Note that $\operatorname{Aff}\left(D_{w}\right)$ acts by quasi-isometries of the $x y$-metric on $D_{w}$. There is a canonical quasi-isometry from $D_{w}$ to $D_{0}$ : restrict the canonical horizontal respecting quasi-isometry $Q_{\gamma_{w}} \rightarrow P_{w}$ to the horizontal leaf $D_{w}$, giving a map $D_{w} \rightarrow X_{H}$, and then take a closest point map to $D_{0}$. In this way we obtain an inclusion

$$
\operatorname{Aff}\left(D_{w}\right) \subset \mathrm{QI}\left(D_{0}\right)=\operatorname{QSym}\left(S^{1}\right)
$$

Note that the image of this inclusion is independent of the choice of horizontal leaf $D_{w}$ in $Q_{\gamma_{w}}$, because if we chose another horizontal leaf $D_{w}^{\prime}$ then the vertical flow on $Q_{\gamma_{w}}$ induces an $x y$-affine homeomorphism $D_{w}^{\prime} \rightarrow D_{w}$ in the correct quasi-isometry class.

Recall that we have a short exact sequence

$$
1 \rightarrow \operatorname{Isom}_{+}\left(D_{w}\right) \rightarrow \operatorname{Aff}\left(D_{w}\right) \rightarrow C_{\gamma_{w}} \rightarrow 1
$$

with $C_{\gamma_{w}} \equiv \mathbf{Z}$ or $D_{\infty}$. Under the injection $\operatorname{Aff}\left(D_{w}\right) \hookrightarrow \operatorname{QSym}\left(S^{1}\right)$, the group $\operatorname{Isom}_{+}\left(D_{w}\right)$ is a finite index supergroup of $\pi_{1}(\Sigma)$. We have a quotient orbifold $\mathcal{O}_{w}=D_{w} / \operatorname{Isom}_{+}\left(D_{w}\right)$ with fundamental group $\pi_{1}\left(\mathcal{O}_{w}\right) \approx \operatorname{Isom}_{+}\left(D_{w}\right)$, and associated to the inclusion $\pi_{1}\left(\mathcal{O}_{w}\right) \subset \pi_{1}(\Sigma)$ there is a finite orbifold covering map $\Sigma \rightarrow \mathcal{O}_{w}$.

In the group $\operatorname{QSym}\left(S^{1}\right)$, take the infinite intersection of the groups $\operatorname{Isom}_{+}\left(D_{w}\right)$ over all periodic lines $w$ in the tree $T_{H}$, and note that this group must be a finite index supergroup of $\pi_{1}(\Sigma)$, in fact it is the fundamental group $\pi_{1}\left(\mathcal{O}_{H}\right)$ of an orbifold $\mathcal{O}_{H}$ which $\Sigma$ finitely covers, the smallest orbifold covered by $\Sigma$ which in turn covers each orbifold $\mathcal{O}_{w}$.

$$
\pi_{1}\left(\mathcal{O}_{H}\right)=\bigcap_{\substack{w \subset T_{H} \\ w \text { periodic }}} \operatorname{Isom}_{+}\left(D_{w}\right)
$$

Note that $\mathcal{O}_{H}$ is the smallest subcover of $\Sigma$ such that the Schottky group $H \subset$ $\mathcal{M}(\Sigma)$ descends via the covering map $\Sigma \rightarrow \mathcal{O}_{H}$ to a free subgroup of $\mathcal{M}\left(\mathcal{O}_{H}\right)$. To 
be precise, since $H$ is free we may choose a section $\sigma: H \rightarrow \mathcal{M}(\Sigma, p) \subset \operatorname{QSym}\left(S^{1}\right)$, and the image group $\sigma H$ acting by conjugation on subgroups of $\operatorname{QSym}\left(S^{1}\right)$ permutes the collection of subgroups $\operatorname{Isom}_{+}\left(D_{w}\right)$ and so $\sigma H \subset \operatorname{Aut}\left(\pi_{1}\left(\mathcal{O}_{H}\right)\right)=$ $\mathcal{M}\left(\mathcal{O}_{H}, p\right)$. Projecting to $\mathcal{M}\left(\mathcal{O}_{H}\right)$ we obtain the free subgroup $H^{\prime}$, and a section $\sigma^{\prime}: H^{\prime} \rightarrow \mathcal{M}\left(\mathcal{O}_{H}, p\right)<\operatorname{QSym}\left(S^{1}\right)$ so that $\sigma H=\sigma^{\prime} H^{\prime}$.

Remark. Orbifold mapping class groups obey many of the properties of surface mapping class groups. The group $\mathcal{M}\left(\mathcal{O}_{H}\right)$ is defined as the group of orbifold homeomorphisms of $\mathcal{O}_{H}$ modulo those which are isotopic to the identity through orbifold homeomorphisms. Just as with mapping class groups of surfaces, choosing a generic point $p \in \mathcal{O}_{H}$ we obtain an isomorphism of short exact sequences

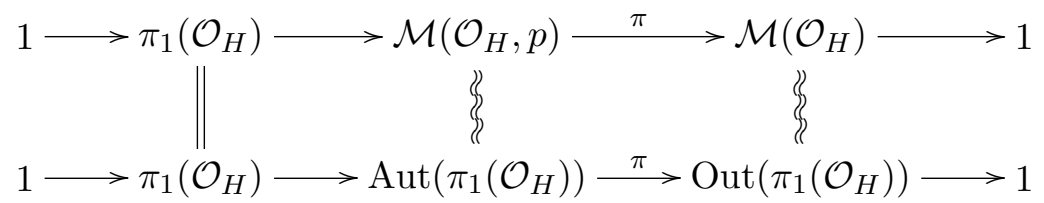

The proof that this isomorphism exists follows the same lines as the proof for surfaces, using the fact that $\pi_{1}\left(\mathcal{O}_{H}\right)$ is centerless.

By choosing a lift of $p$ to the universal cover of $D_{0}=\widetilde{\mathcal{O}}_{H}$ and lifting each element of $\mathcal{M}\left(\mathcal{O}_{H}, p\right)$, we identify $\mathcal{M}\left(\mathcal{O}_{H}, p\right)$ with a subgroup of $\mathrm{QI}\left(D_{0}\right)=\operatorname{QSym}\left(S^{1}\right)$.

In fact $H^{\prime}<\mathcal{M}\left(\mathcal{O}_{H}\right)$ is a Schottky subgroup. To see why, consider the limit set $\Lambda(H) \subset \mathbf{P} \mathcal{M} F(\Sigma)$. Moving into $S^{1}$, the corresponding subset $\left\{\mu_{b} \mid b \in \Lambda(H)\right\}$ of $\mathbf{P} M\left(D S^{1}\right)$ is invariant under $\sigma H$. Notice that for each $b \in \Lambda(H)$, the element $\mu_{b} \in \mathbf{P} M\left(S^{1}\right)$, which is invariant under $\pi_{1}(\Sigma)$, is also invariant under the larger group $\pi_{1}\left(\mathcal{O}_{H}\right)$. When $b$ is an endpoint of a periodic geodesic $w$ in $T_{H}$ this is obvious, because $\mu_{b}$ is invariant under $\pi_{1}\left(\mathcal{O}_{w}\right) \supset \pi_{1}\left(\mathcal{O}_{H}\right)$. But periodic endpoints are dense in $\Lambda(H)$, and so each $\mu_{b}, b \in \Lambda(H)$ is invariant under $\pi_{1}\left(\mathcal{O}_{H}\right)$. We therefore obtain a continuous embedding of $\Lambda(H)$ in $\mathbf{P} \mathcal{M} F\left(\mathcal{O}_{H}\right)$, whose image we denote $\Lambda\left(H^{\prime}\right)$; this embedding has the property that

$$
\left\{\mu_{b} \mid b \in \Lambda(H)\right\}=\left\{\mu_{b^{\prime}} \mid b^{\prime} \in \Lambda\left(H^{\prime}\right)\right\} \quad \text { in } \quad \mathbf{P} M\left(D S^{1}\right)
$$

Moreover, since $\sigma^{\prime} H^{\prime}=\sigma H$, it follows that $\sigma^{\prime} H^{\prime}$ permutes the elements of the above set, and so $\Lambda\left(H^{\prime}\right)$ is in fact invariant under the action of $H^{\prime}$. For any pair $\xi \neq \eta \in \Lambda(H)$ and corresponding pair $\xi^{\prime}, \eta^{\prime} \in \Lambda\left(H^{\prime}\right)$, each $x y$-structure on $\Sigma$ corresponding to a point along the Teichmüller geodesic $\overleftrightarrow{(\xi, \eta)} \subset \mathcal{T}(\Sigma)$ lifts to an $x y$-structures on $D_{0}$ which is invariant under $\pi_{1}\left(\mathcal{O}_{H}\right)$, and we therefore obtain a Teichmüller geodesic $\overleftrightarrow{\left(\xi^{\prime}, \eta^{\prime}\right)}$ in $\mathcal{T}\left(\mathcal{O}_{H}\right)$; the $\pi_{1}\left(\mathcal{O}_{H}\right)$-invariance follows by an 
argument similar to the one just above where we showed invariance for elements $\mu_{b}, b \in \Lambda(H)$. The union of these geodesics over all pairs $\xi^{\prime} \neq \eta^{\prime} \in \Lambda\left(H^{\prime}\right)$ is denoted $\mathcal{H} \Lambda\left(H^{\prime}\right)$ as usual. From this construction we see that the action of $H^{\prime}$ on $\Lambda\left(H^{\prime}\right) \cup \mathcal{H} \Lambda\left(H^{\prime}\right)$ agrees with the action of $H$ on $\Lambda(H) \cup \mathcal{H} \Lambda(H)$. In particular, all the requirements in Theorem 3.1 for $H^{\prime}$ to be convex cocompact with limit set $\Lambda\left(H^{\prime}\right)$ are satisfied, and so $H^{\prime}$ is a Schottky subgroup of $\mathcal{M}\left(\mathcal{O}_{H}\right)$.

Remark. The fact that the orbifold $\mathcal{O}_{H}$ supports a pseudo-Anosov mapping class puts some restrictions on its topology: the underlying surface of $\mathcal{O}_{H}$ must have empty boundary, because otherwise the collection of peripheral curves would be invariant under any mapping class, violating the existence of pseudo-Anosov mapping classes. It follows that $\mathcal{O}_{H}$ is a closed surface with cone singularities.

\subsection{Computing the quasi-isometry group}

In this section we compute $\mathrm{QI}\left(\Gamma_{H}\right)$. Let $\mathcal{C}$ denote the relative commensurator of $H^{\prime}$ in $\mathcal{M}\left(\mathcal{O}_{H}\right)$ :

$$
\mathcal{C}=\operatorname{Comm}_{\mathcal{M}\left(\mathcal{O}_{H}\right)}\left(H^{\prime}\right)
$$

Form the extension group $\Gamma_{\mathcal{C}}$, a subgroup of $\mathcal{M}\left(\mathcal{O}_{H}, p\right)$ as the following diagram shows:

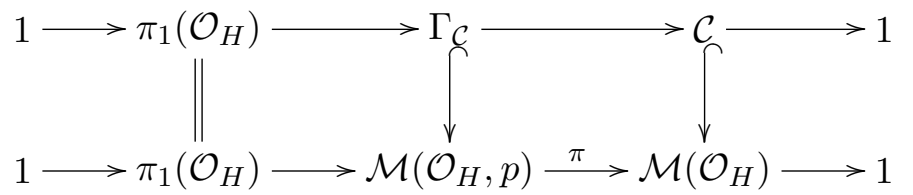

The group $\Gamma_{\mathcal{C}}$ may therefore be regarded as a subgroup of QSym $\left(S^{1}\right)$.

Here is our computation of $\mathrm{QI}\left(\Gamma_{H}\right)$ :

Theorem 7.2. In $\operatorname{QSym}\left(S^{1}\right)$ we have

$$
\mathrm{QI}\left(\Gamma_{H}\right)=\Gamma_{\mathcal{C}}
$$

Moreover, the subgroup $\pi_{1}\left(\mathcal{O}_{H}\right)$ consists of those classes of quasi-isometries of $X_{H}$ which coarsely preserve each horizontal leaf of $X_{H}$.

Proof. To justify this computation, first we show $\mathrm{QI}\left(\Gamma_{H}\right) \subset \Gamma_{\mathcal{C}}$. Consider a quasiisometry $f: X_{H} \rightarrow X_{H}$, regarded as an element of $\operatorname{QSym}\left(S^{1}\right)$; we must show that $f \in \Gamma_{\mathcal{C}}$.

By Lemma 4.3 the quasi-isometry $f$ coarsely respects the horizontal foliation of $X_{H}$ and so $f$ lies over a quasi-isometry of $T_{H}$, also denoted $f$. From Proposition 6.1 it follows that $f$ induces a permutation on the set of periodic hyperplanes of 
$X$ : given a periodic geodesic $w$ in $T_{H}$, if we let $w^{\prime}$ be the geodesic in $T_{H}$ coarsely equivalent to $f(w)$, then $f$ takes $P_{w}$ to $P_{w^{\prime}}$ and $Q_{\gamma_{w}}$ to $Q_{\gamma_{w^{\prime}}}$. It follows that the conjugation action of $f$ on $\operatorname{subgroups}$ of $\operatorname{QSym}\left(S^{1}\right)$ takes $\operatorname{Isom}\left(Q_{\gamma_{w}}\right)=\operatorname{Aff}\left(D_{w}\right)$ to $\operatorname{Isom}\left(Q_{\gamma_{w^{\prime}}}\right)=\operatorname{Aff}\left(D_{w^{\prime}}\right)$. Any isometry of $Q_{\gamma_{w}}$ which preserves each leaf of the horizontal foliation is conjugated by $f$ to a similar isometry of $Q_{\gamma_{w^{\prime}}}$, and so conjugation by $f$ takes $\operatorname{Isom}_{h}\left(Q_{\gamma_{w}}\right)=\operatorname{Isom}_{+}\left(D_{w}\right)$ to $\operatorname{Isom}_{h}\left(Q_{\gamma_{w^{\prime}}}\right)=\operatorname{Isom}_{+}\left(D_{w^{\prime}}\right)$. In other words, conjugation by $f$ preserves the collection of subgroups

$$
\left\{\operatorname{Isom}_{+}\left(D_{w}\right) \mid w \subset T_{H} \text { is periodic }\right\}
$$

Intersecting this collection it follows that conjugation by $f$ in the group $\operatorname{QSym}\left(S^{1}\right)$ preserves the subgroup $\pi_{1}\left(\mathcal{O}_{H}\right)$, acting as an automorphism of that subgroup. In other words, in $\operatorname{QSym}\left(S^{1}\right)$ we have $f \in \operatorname{Aut}\left(\pi_{1}\left(\mathcal{O}_{H}\right)\right)=\mathcal{M}\left(\mathcal{O}_{H}, p\right)$.

Now we show that the image $\pi f \in \mathcal{M}\left(\mathcal{O}_{H}\right)$ lies in $\mathcal{C}=\operatorname{Comm}_{\mathcal{M}\left(\mathcal{O}_{H}\right)}\left(H^{\prime}\right)$. We need the following fact:

Theorem 7.3 (Commensurators of Schottky groups). Let $\mathcal{O}$ be a closed orbifold and $H^{\prime}$ a Schottky subgroup of $\mathcal{M}(\mathcal{O})$. Then the relative commensurator $\operatorname{Comm}_{\mathcal{M}(\mathcal{O})}\left(H^{\prime}\right)$ of $H^{\prime}$ in $\mathcal{M}(\mathcal{O})$ is equal to the subgroup of $\mathcal{M}(\mathcal{O})$ stabilizing the limit set $\Lambda\left(H^{\prime}\right)$, and $H^{\prime}$ has finite index in $\operatorname{Comm}_{\mathcal{M}(\mathcal{O})}\left(H^{\prime}\right)$.

Proof. Let $I$ be the subgroup of $\mathcal{M}(\mathcal{O})$ stabilizing $\Lambda\left(H^{\prime}\right)$, and so $H^{\prime}<I$. Let $\mathcal{H} \Lambda\left(H^{\prime}\right)$ be the weak convex hull of $\Lambda\left(H^{\prime}\right)$ (see Theorem 3.1). Clearly $I$ is also the subgroup of $\mathcal{M}(\mathcal{O})$ stabilizing $\mathcal{H} \Lambda\left(H^{\prime}\right)$. By Theorem 3.1 the group $H^{\prime}$ acts cocompactly on $\mathcal{H} \Lambda\left(H^{\prime}\right)$, and so $I$ acts cocompactly on $\mathcal{H} \Lambda\left(H^{\prime}\right)$. Clearly $I$ acts properly on $\mathcal{H} \Lambda\left(H^{\prime}\right)$. It follows that $H^{\prime}$ has finite index in $I$, which immediately implies $I<\operatorname{Comm}_{\mathcal{M}(\mathcal{O})}\left(H^{\prime}\right)$.

For the opposite inclusion, suppose $\Phi \in \mathcal{M}(\mathcal{O})-I$, and so $\Phi\left(\Lambda\left(H^{\prime}\right)\right) \neq \Lambda\left(H^{\prime}\right)$. The limit set of the Schottky subgroup $\Phi H^{\prime} \Phi^{-1}$ is $\Phi\left(\Lambda\left(H^{\prime}\right)\right)$. Since $\Lambda\left(H^{\prime}\right)$ is closed, $\Phi\left(\Lambda\left(H^{\prime}\right)\right)-\Lambda\left(H^{\prime}\right)$ is open in $\Phi\left(\Lambda\left(H^{\prime}\right)\right)$. Since fixed points of pseudoAnosov elements of $\Phi H^{\prime} \Phi^{-1}$ are dense in $\Phi\left(\Lambda\left(H^{\prime}\right)\right)$, there exists a pseudo-Anosov element $\Psi \in \Phi H^{\prime} \Phi^{-1}$ having a fixed point not in $\Lambda\left(H^{\prime}\right)$. Infinitely many powers of $\Psi$ are therefore not in $H^{\prime}$, and so $H^{\prime} \cap \Phi H^{\prime} \Phi^{-1}$ has infinite index in $\Phi H^{\prime} \Phi^{-1}$.

We showed above that $H^{\prime}$ is a Schottky subgroup of $\mathcal{M}\left(\mathcal{O}_{H}\right)$ and so by Theorem 7.3 it remains to show that $\pi f \in \mathcal{M}\left(\mathcal{O}_{H}\right)$ acting on $\mathbf{P} \mathcal{M} F\left(\mathcal{O}_{H}\right)$ leaves the set $\Lambda\left(H^{\prime}\right)$ invariant. For this purpose it suffices to show that the action of $f \in$ $\operatorname{QSym}\left(S^{1}\right)$ leaves the set $\left\{\mu_{b^{\prime}} \mid b^{\prime} \in \Lambda\left(H^{\prime}\right)\right\}$ invariant. However, we know that this set equals $\left\{\mu_{b} \mid b \in \Lambda(H)\right\}$, and we also know that $f$ leaves this set invariant: $f$ permutes the elements of this set which are endpoints of periodic geodesics in $T_{H}$, by Proposition 6.1, but the endpoints of periodic geodesics are dense. 
This completes the proof of the inclusion $\mathrm{QI}\left(\Gamma_{H}\right) \subset \Gamma_{\mathcal{C}}$.

For the opposite inclusion, consider the following commutative diagram of short exact sequences:

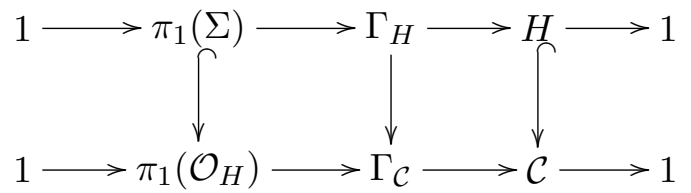

The left vertical arrow is a finite index injection because $\Sigma \rightarrow \mathcal{O}_{H}$ is a finite covering. The right vertical arrow is a finite index injection by Theorem 7.3 using the isomorphism $H \approx H^{\prime}$. It follows that the middle vertical arrow is a finite index injection. But this shows that $\Gamma_{\mathcal{C}}$ is a finite index supergroup of $\Gamma_{H}$ in $\operatorname{QSym}\left(S^{1}\right)$, and so $\Gamma_{\mathcal{C}} \subset \mathrm{QI}\left(\Gamma_{H}\right)$.

This completes the computation of $\mathrm{QI}\left(\Gamma_{H}\right)$.

To complete the proof of Theorem 7.2, it remains to prove that an element of $\mathrm{QI}\left(\Gamma_{H}\right)=\Gamma_{\mathcal{C}}$ is in the normal subgroup $\pi_{1}\left(\mathcal{O}_{H}\right)$ if and only if it coarsely preserves each horizontal leaf of $X_{H}$. Evidently $\pi_{1}\left(\mathcal{O}_{H}\right)$ coarsely preserves each horizontal leaf. Suppose conversely that the quasi-isometry $f: X_{H} \rightarrow X_{H}$ coarsely preserves each horizontal leaf; let $[f] \in \mathrm{QI}\left(\Gamma_{H}\right)$ be the coarse equivalence class of $f$. It follows that $f$ coarsely respects each hyperplane of $X_{H}$, in particular each periodic hyperplane $P_{w}$, and so we have $[f] \in \operatorname{Aff}\left(D_{w}\right)$ for each periodic line $w$ in $T_{H}$. But we can say more, as follows. The inclusion map $\Gamma_{H} \rightarrow \mathrm{QI}\left(\Gamma_{H}\right)$, being injective and with finite index image, is a quasi-isometry, and we therefore obtain a quasi-isometry $\mathrm{QI}\left(\Gamma_{H}\right) \rightarrow X_{H}$. Using this quasi-isometry we may conjugate the left action of $\mathrm{QI}\left(\Gamma_{H}\right)$ on itself to obtain a quasi-action of $\mathrm{QI}\left(\Gamma_{H}\right)$ on $X_{H}$. In particular, we obtain a sequence of uniform quasi-isometries $f_{n}: X_{H} \rightarrow X_{H}$, with $\left[f_{n}\right]=[f]^{n}$. Each of the $f_{n}$ coarsely preserves each horizontal leaf of $X_{H}$, and note that the coarseness constant is uniform independent of $n$ and of the leaf, by application of Proposition 4.1 using uniformity of the quasi-isometry constants of $f_{n}$. It follows that each $f_{n}$ coarsely preserves each periodic hyperplane $P_{w}$, and $f_{n}$ coarsely preserves each horizontal leaf of $P_{w}$, again with uniform coarseness constants independent of $n$. This implies that $[f] \in \operatorname{Isom}_{+}\left(D_{w}\right)$. We therefore have

$$
[f] \in \bigcap_{w} \operatorname{Isom}_{+}\left(D_{w}\right)=\pi_{1}\left(\mathcal{O}_{H}\right)
$$




\subsection{Computing the commensurator group}

In this section we use our knowledge of quasi-isometries of $\Gamma_{H}$ to compute the commensurator of $\Gamma_{H}$ and complete the proof of Theorem 1.3. This is the first instance we know of where this technique of computing a commensurator group is used. We prove, in Theorem 7.4, that when the left multiplication homomorphism $\Gamma \rightarrow \mathrm{QI}(\Gamma)$ is an injection with finite index image, the natural homomorphism $i: \operatorname{Comm}(\Gamma) \rightarrow \mathrm{QI}(\Gamma)$ is an isomorphism. Combining with Theorem 7.2 we obtain the desired computation of $\operatorname{Comm}\left(\Gamma_{H}\right)$. We do not have a proof of the computation of $\operatorname{Comm}\left(\Gamma_{H}\right)$ without going through all of the work required to understand quasiisometries; a purely algebraic computation of $\operatorname{Comm}\left(\Gamma_{H}\right)$ would be interesting.

For any finitely-generated group $\Gamma$, a commensuration $\phi: G_{1} \rightarrow G_{2}$ extends to a quasi-isometry of $\Gamma$ by precomposing $\phi$ with a closest point projection $\Gamma \rightarrow G_{1}$ and postcomposing with inclusion $G_{2} \rightarrow \Gamma$. The coarse equivalence class of this extension is well-defined, giving a natural homomorphism $i$ : $\operatorname{Comm}(\Gamma) \rightarrow \operatorname{QI}(\Gamma)$. Note that we have a commutative triangle

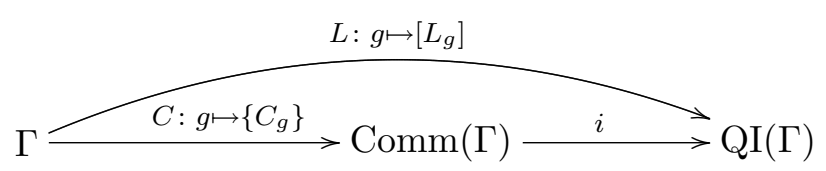

where $L_{g}$ is the left multiplication map $x \rightarrow g x$ with coarse equivalence class $\left[L_{g}\right] \in$ $\mathrm{QI}(\Gamma)$, and $C_{g}$ is the conjugation automorphism $x \rightarrow g x g^{-1}$ with equivalence class $\left\{C_{g}\right\} \in \operatorname{Comm}(\Gamma)$; commutativity follows because right multiplication by $g^{-1}$ moves each point in $\Gamma$ a bounded amount.

Theorem 7.4. Given a finitely generated group $\Gamma$, if $L: \Gamma \rightarrow \mathrm{QI}(\Gamma)$ is an injection with finite index image, then $i: \operatorname{Comm}(\Gamma) \rightarrow \mathrm{QI}(\Gamma)$ is an isomorphism.

This theorem, proved in collaboration Kevin Whyte, is broken into three steps:

(1) injectivity of $i: \operatorname{Comm}(\Gamma) \rightarrow \mathrm{QI}(\Gamma)$;

(2) construction of an injective map $\Psi: \mathrm{QI}(\Gamma) \rightarrow \operatorname{Comm}(\Gamma)$;

(3) the proof that $\Psi \circ i$ is the identity on $\operatorname{Comm}(\Gamma)$.

\section{Step 1: Injectivity of $i$.}

Proposition 7.5 (K. Whyte). If $\Gamma$ is any finitely generated group then the natural map $i: \operatorname{Comm}(\Gamma) \rightarrow \mathrm{QI}(\Gamma)$ is injective. 
Proof. Suppose $\phi: G_{1} \rightarrow G_{2}$ is a commensuration of $\Gamma$ such that $i(\phi)$ equals the identity in $\mathrm{QI}(\Gamma)$. Then there is a bounded function $\delta: \Gamma \rightarrow \Gamma$ so that for all $g \in G_{1}$ we have

$$
\phi(g)=g \delta(g)
$$

Since $\delta$ is bounded, the cardinality $M=\#$ image $(\delta)$ is finite.

Plugging the above equation into $\phi(g h)=\phi(g) \phi(h)$ gives

$$
h^{-1} \delta(g) h=\delta(g h) \delta(h)^{-1}
$$

Note that this is true for all $h \in G_{1}$, and the right hand side takes on at most $M^{2}$ values. This implies that the centralizer of $\delta(g)$ in $G_{1}$ has index at most $M^{2}$. Intersecting all subgroups of $G_{1}$ of index $\leq M^{2}$ gives a finite index subgroup $H<G_{1}$ which commutes with each $\delta(g)$. From the above equation it follows that $\delta$ is a homomorphism on $H$. Since image $(\delta)$ is finite it follows that $\delta$ has finite index $\operatorname{kernel} \operatorname{ker}(\delta)$ in $H$, and so $\operatorname{ker}(\delta)$ has finite index in $G_{1}$ and in $\Gamma$. In other words, $\phi(g)=g$ on the finite index subgroup $\operatorname{ker}(\delta)$ of $\Gamma$, and so $\phi$ represents the identity element of $\operatorname{Comm}(\Gamma)$.

For any finitely generated group $\Gamma$ the kernel of $C: \Gamma \rightarrow \operatorname{Comm}(\Gamma)$ is the virtual center $\mathrm{VZ}(\Gamma)$ consisting of all $g \in \Gamma$ whose centralizer has finite index in $\Gamma$. Together with Proposition 7.5 it follows that injectivity of $L: \Gamma \rightarrow \mathrm{QI}(\Gamma)$ is equivalent to the triviality of $\mathrm{VZ}(\Gamma)$.

Step 2: An injection $\Psi: \mathrm{QI}(\Gamma) \rightarrow \operatorname{Comm}(\Gamma)$. Identifying $\Gamma$ with its image $L(\Gamma)<\mathrm{QI}(\Gamma)$, a finite index subgroup, it follows that any automorphism of $\mathrm{QI}(\Gamma)$ restricts to a commensuration of $\Gamma$. In particular, given $F \in \mathrm{QI}(\Gamma)$, the inner automorphism $G \mapsto F G F^{-1}$ of $\mathrm{QI}(\Gamma)$ restricts to a commensuration $\Psi_{F}$ of $\Gamma$, giving a well-defined homomorphism $\Psi: \mathrm{QI}(\Gamma) \rightarrow \operatorname{Comm}(\Gamma)$. Keeping in mind the definition of $L: \Gamma \rightarrow \mathrm{QI}(\Gamma)$, this means that for each $F \in \mathrm{QI}(\Gamma)$ and each $x \in \Gamma$ we have the following equation in $\mathrm{QI}(\Gamma)$ :

$$
F \cdot\left[L_{x}\right] \cdot F^{-1}=\left[L_{\Psi_{F}(x)}\right]
$$

To prove that $\Psi$ is an injection, given $F=[f] \in \mathrm{QI}(\Gamma)$ suppose that $\Psi_{F}$ is the identity map when restricted to a finite index subgroup $G_{1}$ of $\Gamma$. Then for all $x \in G_{1}$ we have

$$
\begin{aligned}
F \cdot\left[L_{x}\right] \cdot F^{-1} & =\left[L_{x}\right] \\
F \cdot\left[L_{x}\right] & =\left[L_{x}\right] \cdot F \\
{\left[f \circ L_{x}\right] } & =\left[L_{x} \circ f\right]
\end{aligned}
$$


which means that there exists a bounded function $\delta: \Gamma \rightarrow \Gamma$ such that

$$
f(x y)=x f(y) \delta(y), \quad x, y \in G_{1}
$$

Plugging in $y=1$ we get

$$
f(x)=x f(1) \delta(1), \quad x \in G_{1}
$$

This shows that the function $f$ is a bounded distance from the identity map on $G_{1}$, and so $F$ is the identity element in $\mathrm{QI}(\Gamma)$.

Step 3: $\Psi \circ i: \operatorname{Comm}(\Gamma) \rightarrow \operatorname{Comm}(\Gamma)$ is the identity. Given a commensuration $\phi$ of $\Gamma$, we obtain a commensuration $\phi^{\prime}=\Psi_{i(\phi)}$ satisfying the formulas

$$
\begin{aligned}
{\left[\phi \circ L_{x} \circ \phi^{-1}\right] } & =\left[L_{\phi^{\prime}(x)}\right] \\
{\left[\phi \circ L_{x} \circ \phi^{-1} \circ L_{\phi^{\prime}\left(x^{-1}\right)}\right] } & =[\mathrm{Id}]
\end{aligned}
$$

for all $x$ in a certain finite index subgroup of $\Gamma$. We must prove that $\phi$ and $\phi^{\prime}$ agree on a further finite index subgroup of $\Gamma$.

From the above equation it follows that there is a bounded function $\delta: \Gamma \rightarrow \Gamma$ such that for all $h$ in a certain finite index subgroup $H$ of $\Gamma$ we have:

$$
\begin{aligned}
\phi\left(x \cdot \phi^{-1}\left(\phi^{\prime}\left(x^{-1}\right) \cdot h\right)\right. & =h \cdot \delta(h) \\
\phi(x) \cdot \phi^{\prime}\left(x^{-1}\right) \cdot h & =h \cdot \delta(h) \\
h^{-1} \cdot\left(\phi(x) \cdot \phi^{\prime}\left(x^{-1}\right)\right) \cdot h & =\delta(h)
\end{aligned}
$$

This shows that $\phi(x) \cdot \phi^{\prime}\left(x^{-1}\right)$ has only finitely many conjugates by elements of $H$, and so the centralizer of $\phi(x) \cdot \phi^{\prime}\left(x^{-1}\right)$ in $H$ has finite index in $H$. It follows that the centralizer of $\phi(x) \cdot \phi^{\prime}\left(x^{-1}\right)$ in $\Gamma$ has finite index in $\Gamma$, which by definition of $\operatorname{VZ}(\Gamma)$ gives $\phi(x) \cdot \phi^{\prime}\left(x^{-1}\right) \in \mathrm{VZ}(\Gamma)$. But since $L: \Gamma \rightarrow \mathrm{QI}(\Gamma)$ is injective, the virtual center $\operatorname{VZ}(\Gamma)$ is trivial, showing that $\phi(x)=\phi^{\prime}(x)$ for all $x$ in a finite index subgroup of $\Gamma$.

Combining steps $1-3$ it follows that $\Psi: \mathrm{QI}(\Gamma) \rightarrow \operatorname{Comm}(\Gamma)$ is surjective, and so $\Psi$ is an isomorphism with inverse $i: \operatorname{Comm}(\Gamma) \rightarrow \mathrm{QI}(\Gamma)$, finishing the proof of Theorem 7.4.

Remark. The proof of Theorem 7.4 yields a more general conclusion: if $\Gamma$ is a finitely generated group whose virtual center is trivial, then $\operatorname{Comm}(\Gamma)$ is isomorphic to the relative commensurator of $L(\Gamma)$ in $\mathrm{QI}(\Gamma)$. The condition that the virtual center be trivial cannot be dropped: for example, a finite group has trivial quasi-isometry group but rarely is its abstract commensurator group trivial. 


\subsection{Proving Theorem 1.1 and Theorem 1.2}

Theorem 1.2 follows immediately from Theorem 1.3 by a standard technique (see, e.g. [Sch96]). The basic observation we need says that if $\Gamma$ is a finitely generated group and if the homomorphism $\Gamma \rightarrow \mathrm{QI}(\Gamma)$ has finite cokernel and kernel, then for any finitely generated group $H$ and any quasi-isometry $\phi: H \rightarrow \Gamma$ the induced homomorphism $\phi_{*}: H \rightarrow \mathrm{QI}(\Gamma)$ has finite index kernel and cokernel. As a consequence, the groups $H$ and $\Gamma$ are weakly commensurable, because their images are commensurable in $\mathrm{QI}(\Gamma)$.

We now prove Theorem 1.1. Let $H_{i}<\mathcal{M}\left(\Sigma_{g_{i}}\right), i=1$, 2, be Schottky groups of rank $\geq 2$ with $g_{i} \geq 2$. We must prove the equivalence of the following four statements:

(1) $\Gamma_{H_{1}}$ and $\Gamma_{H_{2}}$ are quasi-isometric.

(2) $\Gamma_{H_{1}}$ and $\Gamma_{H_{2}}$ are abstractly commensurable.

(3) There is an isomorphism $\mathcal{O}_{H_{1}} \approx \mathcal{O}_{H_{2}}$ such that in the group $\mathcal{M}\left(\mathcal{O}_{H_{1}}\right)=$ $\mathcal{M}\left(\mathcal{O}_{H_{2}}\right)$ the Schottky subgroups $H_{1}$ and $H_{2}$ are commensurable, meaning that $H_{1} \cap H_{2}$ has finite index in each of $H_{1}$ and $H_{2}$.

(4) There is an isomorphism $\mathcal{O}_{1} \approx \mathcal{O}_{2}$ such that in the group $\mathcal{M}\left(\mathcal{O}_{1}\right)=\mathcal{M}\left(\mathcal{O}_{2}\right)$ the Schottky groups $H_{1}$ and $H_{2}$ have the same limit set in the Thurston boundary of the Teichmüller space $\mathcal{T}\left(\mathcal{O}_{1}\right)=\mathcal{T}\left(\mathcal{O}_{2}\right)$.

We also add in a fifth equivalent statement:

(5) The groups $\mathrm{QI}\left(\Gamma_{H_{1}}\right)$ and $\mathrm{QI}\left(\Gamma_{H_{2}}\right)$ are isomorphic.

The equivalence of statements (1) and (2) and (5) follows immediately from Theorem 1.3 using the fact that a quasi-isometry between two groups induces an isomorphism between their quasi-isometry groups.

The fact that (3) implies (2) is an immediate consequence of the commutative diagram 7.1 applied to $\Gamma_{H_{1}}$ and to $\Gamma_{H_{2}}$.

To prove that (1) implies (3), suppose that there is a quasi-isometry $\Gamma_{H_{1}} \rightarrow$ $\Gamma_{H_{2}}$, which induces an isomorphism of quasi-isometry groups $\mathrm{QI}\left(\Gamma_{H_{1}}\right) \approx \mathrm{QI}\left(\Gamma_{H_{2}}\right)$. Consider, for each $i=1,2$, the short exact sequence

$$
1 \rightarrow \pi_{1}\left(\mathcal{O}_{i}\right) \rightarrow \mathrm{QI}\left(\Gamma_{H_{i}}\right) \rightarrow \mathcal{C}_{i} \rightarrow 1
$$

where as usual $\mathcal{O}_{i}=\mathcal{O}_{H_{i}}$ is the smallest orbifold subcover of $\Sigma_{g_{i}}$ to which $H_{i}$ descends, the subgroup $\mathcal{C}_{i}<\mathcal{M}\left(\mathcal{O}_{i}\right)$ is the relative commensurator of $H_{i}$ in $\mathcal{M}\left(\mathcal{O}_{i}\right)$, and $H_{i}$ has finite index in $\mathcal{C}_{i}$. 
We claim that the isomorphism between $\mathrm{QI}\left(\Gamma_{H_{1}}\right)$ and $\mathrm{QI}\left(\Gamma_{H_{2}}\right)$ must take $\pi_{1}\left(\mathcal{O}_{1}\right)$ to $\pi_{1}\left(\mathcal{O}_{2}\right)$. This provides an isomorphism $\mathcal{O}_{1} \approx \mathcal{O}_{2}$ such that the induced isomorphism $\mathcal{M}\left(\mathcal{O}_{1}\right) \approx \mathcal{M}\left(\mathcal{O}_{2}\right)$ takes $\mathcal{C}_{1}$ to $\mathcal{C}_{2}$, and statement (3) immediately follows.

To prove the claim, consider the model space $X_{H_{i}}$ with its horizontal foliation. By Theorem 7.2 the subgroup $\pi_{1}\left(\mathcal{O}_{i}\right)$ of $\mathrm{QI}\left(\Gamma_{H_{i}}\right) \approx \mathrm{QI}\left(X_{H_{i}}\right)$ consists of quasiisometries which coarsely preserve each leaf of the horizontal foliation. The quasiisometry $X_{H_{1}} \rightarrow X_{H_{2}}$ coarsely respects horizontal foliations by Lemma 4.3 , and so the coarse leaf preserving elements of $\mathrm{QI}\left(X_{H_{1}}\right)$ are taken bijectively by the isomorphism $\mathrm{QI}\left(X_{H_{1}}\right) \leftrightarrow \mathrm{QI}\left(X_{H_{2}}\right)$ to the coarse leaf respecting quasi-isometries of $X_{H_{2}}$. In other words, $\pi_{1}\left(\mathcal{O}_{1}\right)$ is taken to $\pi_{1}\left(\mathcal{O}_{2}\right)$.

Finally, the equivalence of (3) and (4) follows immediately from Theorem 7.3, completing the proof of Theorem 1.1.

\section{Closing remarks}

\subsection{Surfaces versus orbifolds}

As remarked in the introduction, the universe of groups quasi-isometric to (orbifold)by-(virtual Schottky) groups is exactly the same as the universe of groups quasiisometric to (surface)-by-(Schottky) groups:

Proposition 8.1. Given a closed orbifold $\mathcal{O}$ and a virtual Schottky subgroup $N<$ $\mathcal{M}(\mathcal{O})$, consider the extension $\Gamma_{N}$ defined by

$$
1 \rightarrow \pi_{1}(\mathcal{O}) \rightarrow \Gamma_{N} \rightarrow N \rightarrow 1
$$

There exists a closed oriented surface $\Sigma$ of genus $g \geq 2$, and a Schottky subgroup $H<\mathcal{M}(\Sigma)$, such that the group $\Gamma_{H}=\pi_{1}(\Sigma) \rtimes H$ has finite index in $\Gamma_{N}$, and so the two groups are quasi-isometric.

Proof. There is a Schottky subgroup $H^{\prime}<N$ of finite index; it follows that $\Gamma_{H^{\prime}}$ has finite index in $\Gamma_{N}$. Choose a splitting $H^{\prime} \rightarrow \mathcal{M}(\mathcal{O}, p)$, consider the action of $H^{\prime}$ on $\pi_{1}(\mathcal{O})$ by automorphisms. Choose a finite surface cover $\Sigma^{\prime}$ with corresponding

subgroup $\pi_{1}\left(\Sigma^{\prime}\right)<\pi_{1}(\mathcal{O})$, and consider the orbit of $\pi_{1}\left(\Sigma^{\prime}\right)$ under the action of $H^{\prime}$. This orbit consists of a finite collection of finite index subgroups of $\pi_{1}(\mathcal{O})$, whose intersection is a finite index subgroup corresponding to a surface group $\Sigma$ which is a cover of $\Sigma^{\prime}$. The group $H^{\prime}<\mathcal{M}(\mathcal{O}, p)$ lifts to a subgroup $H<\mathcal{M}(\Sigma, p)$, which projects to a Schottky subgroup of $\mathcal{M}(\Sigma)$. The group $\Gamma_{H}=\pi_{1}(\Sigma) \rtimes H$ is therefore a (surface)-by-(Schottky) group with finite index in $\Gamma_{H^{\prime}}$, and so also in the original group $\Gamma_{N}$. 


\subsection{Fibered hyperbolic 3-manifold groups}

As mentioned in the footnote on page 3, the method of proof of Theorem 1.1 shows that if two word hyperbolic surface-by-free groups are quasi-isometric then they are horizontal respecting quasi-isometric, indeed they are horizontal respecting commensurable, as long as the free group has rank $\geq 2$. When the free group is infinite cyclic this fails: all hyperbolic 3-manifolds fibering over the circle have quasi-isometric fundamental groups; but there exist fibered hyperbolic 3-manifolds which are not abstract commensurable - take, for example, an arithmetic example and a non-arithmetic example.

Nevertheless, we do obtain a classification of fundamental groups of fibered hyperbolic 3-manifold groups up to horizontal respecting quasi-isometry. Namely, let $\Sigma$ be a closed surface of genus $g \geq 2$, let $\psi \in \mathcal{M}(\Sigma)$ be a pseudo-Anosov mapping class generating an infinite cyclic subgroup $\langle\psi\rangle$ of $\mathcal{M}(\Sigma)$, and let $\Gamma_{\langle\psi\rangle}$ be the associated extension group of $\pi_{1}(\Sigma)$ by $\langle\psi\rangle$. Let $\mathcal{O}_{\langle\psi\rangle}$ be the smallest subcover of $\Sigma$ to which $\langle\psi\rangle$ descends, let $\mathcal{C}_{\langle\psi\rangle}$ be the relative commensurator of $\langle\psi\rangle$ in $\mathcal{M}\left(\mathcal{O}_{\langle\psi\rangle}\right)$, and let $\Gamma_{\mathcal{C}_{\langle\psi\rangle}}$ be the extension of $\pi_{1}\left(\mathcal{O}_{\langle\psi\rangle}\right)$ by $\mathcal{C}_{\langle\psi\rangle}$. Note that $\mathcal{C}_{\langle\psi\rangle}$ contains $\langle\psi\rangle$ with finite index by [BLM83], and so $\Gamma_{C_{\langle\psi\rangle}}$ contains $\Gamma_{\langle\psi\rangle}$ with finite index. The proofs of $\S 7$ now apply directly to obtain the following classification theorem, which parallels Theorem 1.1:

Theorem 8.2. Given closed surfaces $\Sigma_{i}, i=1,2$, and pseudo-Anosov mapping classes $\psi_{i}$ of $\mathcal{M}\left(\Sigma_{i}\right)$, the following are equivalent, where $\mathcal{O}_{i}=\mathcal{O}_{\left\langle\psi_{i}\right\rangle}$, etc.:

(1) There exists a horizontal respecting quasi-isometry $\Gamma_{\left\langle\psi_{1}\right\rangle} \rightarrow \Gamma_{\left\langle\psi_{2}\right\rangle}$.

(2) The groups $\Gamma_{\left\langle\psi_{1}\right\rangle}, \Gamma_{\left\langle\psi_{2}\right\rangle}$ are horizontal respecting commensurable, meaning that there is an isomorphism from a finite index subgroup $H_{1}<\Gamma_{\left\langle\psi_{1}\right\rangle}$ to a finite index subgroup $H_{2}<\Gamma_{\left\langle\psi_{2}\right\rangle}$, taking $H_{1} \cap \pi_{1}\left(\mathcal{O}_{1}\right)$ to $H_{2} \cap \pi_{1}\left(\mathcal{O}_{2}\right)$.

(3) There is an isomorphism $\mathcal{O}_{1} \approx \mathcal{O}_{2}$ such that in the group $\mathcal{M}\left(\mathcal{O}_{1}\right)=\mathcal{M}\left(\mathcal{O}_{2}\right)$ the mapping classes $\psi_{1}$ and $\psi_{2}$ have equal powers.

(4) There is an isomorphism $\mathcal{O}_{1} \approx \mathcal{O}_{2}$ such that in the group $\mathcal{M}\left(\mathcal{O}_{1}\right)=\mathcal{M}\left(\mathcal{O}_{2}\right)$ the mapping classes $\psi_{1}$ and $\psi_{2}$ have the same limit set in the Thurston boundary of the Teichmüller space $\mathcal{T}\left(\mathcal{O}_{1}\right)=\mathcal{T}\left(\mathcal{O}_{2}\right)$, i.e. they have the same stable/unstable measured foliation pairs.

(5) There is an isomorphism $\Gamma_{\mathcal{C}_{1}} \approx \Gamma_{\mathcal{C}_{2}}$ taking $\pi_{1}\left(\mathcal{O}_{1}\right)$ to $\pi_{1}\left(\mathcal{O}_{2}\right)$. 
It is easy to use this theorem to obtain infinitely many distinct horizontal respecting quasi-isometry classes of groups $\Gamma_{H}$, for cyclic pseudo-Anosov groups $H$. This should be contrasted with the fact that all of the groups $\Gamma_{H}$ are quasiisometric to each other and to $\mathbf{H}^{3}$, by Thurston's hyperbolization theorem for fibered 3-manifolds [Ota96].

\section{References}

[AB56] L. Ahlfors and A. Beurling, The boundary correspondence under quasiconformal mappings, Acta Math. 96 (1956), 125-142.

[Abi80] W. Abikoff, The real analytic theory of Teichmüller space, Lecture Notes in Mathematics, vol. 820, Springer, 1980.

[Ber78] L. Bers, An extremal problem for quasiconormal mappings and a theorem by Thurston, Acta Math. 141 (1978), 73-98.

[BH99] M. Bridson and A. Haefliger, Metric spaces of non-positive curvature, Springer, 1999.

[BLM83] J. Birman, A. Lubotzky, and J. McCarthy, Abelian and solvable subgroups of the mapping class group, Duke Math. J. 50 (1983), no. 4, 1107-1120.

[Bou00] M. Bourdon, Sur les immeubles fuchsiens et leur type de quasiisométrie, Ergodic Th. \& Dyn. Sys. 20 (2000), no. 2, 343-364.

[Far97] B. Farb, The quasi-isometry classification of lattices in semisimple Lie groups, Math. Res. Letters 4 (1997), no. 5, 705-717.

$\left[\right.$ FLP $\left.^{+} 79\right]$ A. Fathi, F. Laudenbach, V. Poenaru, et al., Travaux de Thurston sur les surfaces, Astérisque, vol. 66-67, Société Mathématique de France, 1979.

[FM98] B. Farb and L. Mosher, A rigidity theorem for the solvable BaumslagSolitar groups, Invent. Math. 131 (1998), no. 2, 419-451, Appendix by D. Cooper.

[FM99] B. Farb and L. Mosher, Quasi-isometric rigidity for the solvable Baumslag-Solitar groups, II, Invent. Math. 137 (1999), no. 3, 613-649.

[FM00a] B. Farb and L. Mosher, On the asymptotic geometry of abelian-by-cyclic groups, Acta Math. 184 (2000), no. 2, 145-202. 
[FM00b] B. Farb and L. Mosher, Schottky subgroups of mapping class groups, in preparation, 2000.

[FM00c] S. Fenley and L. Mosher, Quasigeodesic flows in hyperbolic threemanifolds, Topology (2000), to appear.

[GM91] F. Gardiner and H. Masur, Extremal length geometry of Teichmüller space, Complex Variables Theory Appl. 16 (1991), 209-237.

[Han85] M. Handel, Global shadowing of pseudo-Anosovs, Ergodic Th. \& Dyn. Sys. 5 (1985), 373-377.

[Han88] M. Handel, Entropy and semi-conjugacy in dimension two, Ergodic Th. \& Dyn. Sys. 8 (1988), 585-596.

[HM79] J. Hubbard and H. Masur, Quadratic differentials and foliations, Acta Math. 142 (1979), 221-274.

[IT92] Y. Imayoshi and M. Taniguchi, An introduction to Teichmüller spaces, Springer, 1992.

[KK98] M. Kapovich and B. Kleiner, Hyperbolic groups with 1-dimensional boundary, preprint, 1998.

[Mar91] G. Margulis, Discrete subgroups of semisimple Lie groups, Springer, 1991.

[Mas80] H. Masur, Uniquly ergodic quadratic differentials, Comment. Math. Helv. 55 (1980), 25-266.

[Mil68] J. Milnor, A note on curvature and fundamental group, J. Diff. Geom. 2 (1968), 1-7.

[Min96] Y. Minsky, Quasi-projections in Teichmüller space, J. Reine Angew. Math. 473 (1996), 121-136.

[Mit98] M. Mitra, Cannon-Thurston maps for trees of hyperbolic metric spaces, J. Diff. Geom. 48 (1998), 135-164.

[Mos89] L. Mosher, Equivariant spectral decomposition for flows with a $\mathbf{Z}$-action, Ergodic Th. \& Dyn. Sys. 9 (1989), 329-378.

[Mos96] L. Mosher, Hyperbolic extensions of groups, J. Pure and Appl. Alg. 110 (1996), no. 3, 305-314. 
[Mos97] L. Mosher, A hyperbolic-by-hyperbolic hyperbolic group, Proc. AMS 125 (1997), no. 12, 3447-3455.

[MSW00] L. Mosher, M. Sageev, and K. Whyte, Quasi-actions on trees II: BassSerre trees, in preparation, 2000.

[Ota96] J.-P. Otal, Le théorème d'hyperbolisation pour les variétés fibrées de dimension 3, Astérisque, no. 235, Société Mathématique de France, 1996.

[Roy71] H. Royden, Automorphisms and isometries of Teichmüller space, Advances in the Theory of Riemann Surfaces (Proc. Conf., Stony Brook, N.Y., 1969), Ann. of Math. Studies, vol. 66, 1971, pp. 369-383.

[Sch96] R. Schwartz, The quasi-isometry classification of rank one lattices, IHES Sci. Publ. Math. 82 (1996).

[Sch97] R. Schwartz, Symmetric patterns of geodesics and automorphisms of surface groups, Invent. Math. 128 (1997), 177-199.

[Thu97] W. P. Thurston, Three-manifolds, foliations and circles, I, Preliminary version, Dec. 291997.

[Zim84] R. Zimmer, Ergodic theory and semisimple groups, Monographs in Math, vol. 81, Birkhauser, 1984.

Benson Farb:

Department of Mathematics, Univeristy of Chicago

5734 University Ave.

Chicago, Il 60637

E-mail: farb@math.uchicago.edu

Lee Mosher:

Department of Mathematics, Rutgers University, Newark

Newark, NJ 07102

E-mail: mosher@andromeda.rutgers.edu 